\title{
Fitting the Viking lander surface pressure cycle with a Mars General Circulation Model
}

\author{
Xin Guo, ${ }^{1}$ W. Gregory Lawson, ${ }^{1}$ Mark I. Richardson,,${ }^{1,2}$ and Anthony Toigo ${ }^{3}$ \\ Received 14 November 2008; revised 18 March 2009; accepted 21 May 2009; published 30 July 2009.
}

[1] We present a systematic attempt to fit the Viking lander surface pressure cycle using a Mars General Circulation Model, MarsWRF. Following the earlier study by Wood and Paige (1992) using a one-dimensional model, high-precision fitting was achieved by tuning five time-independent parameters: the albedo and emissivity of the seasonal caps of the two hemispheres and the total $\mathrm{CO}_{2}$ inventory in the atmosphere frost system. We used a linear iterative method to derive the best fit parameters: albedo of the northern cap $=0.795$, emissivity of the northern cap $=0.485$, albedo of the southern cap $=0.461$, emissivity of the southern cap $=0.785$, and total $\mathrm{CO}_{2}$ mass $=2.83 \times 10^{16} \mathrm{~kg}$. If these parameters are used in MarsWRF, the smoothed surface pressure residual at the VL1 site is always smaller than several Pascal through a year. As in other similar studies, the best fit parameters do not match well with the current estimation of the seasonal cap radiative properties, suggesting that important physics contributing to the energy balance not explicitly included in MarsWRF have been effectively aliased into the derived parameters. One such effect is likely the variation of thermal conductivity with depth in the regolith due to the presence of water ice. Including such a parameterization in the fitting process improves the reasonableness of the best fit cap properties, mostly improving the emissivities. The conductivities required in the north to provide the best fit are higher than those required in the south. A completely physically reasonable set of fit parameters could still not be attained. Like all prior published GCM simulations, none of the cases considered are capable of predicting a residual southern $\mathrm{CO}_{2}$ cap.

Citation: Guo, X., W. G. Lawson, M. I. Richardson, and A. Toigo (2009), Fitting the Viking lander surface pressure cycle with a Mars General Circulation Model, J. Geophys. Res., 114, E07006, doi:10.1029/2008JE003302.

\section{Introduction}

[2] In every atmospheric General Circulation Model (GCM), one of the first and foremost issues to consider is the total mass of the atmosphere. Its value affects all subsequent calculations performed in the GCM, including dynamics, radiative transfer, tracer and energy transport, chemical reactions, etc. When a component of the atmosphere is volatile (changes state for commonly encountered environmental conditions), the surface pressure may be affected. For the Earth, where the abundance of water vapor is modestly high, many GCMs include the varying contribution of water vapor partial pressure when calculating the full surface pressure. For planets where their major atmospheric constituents condense (such as Mars, Triton, and Pluto), consideration of the role of phase change in varying the surface pressure is important.

\footnotetext{
${ }^{1}$ Division of Geological and Planetary Sciences, California Institute of Technology, Pasadena, California, USA.

${ }^{2}$ Ashima Research, Pasadena, California, USA.

${ }^{3}$ Center for Radiophysics and Space Research, Cornell University, Ithaca, New York, USA.

Copyright 2009 by the American Geophysical Union. 0148-0227/09/2008JE003302\$09.00
}

[3] Carbon dioxide $\left(\mathrm{CO}_{2}\right)$ is the leading gaseous species on Mars, comprising $95 \%$ of the atmosphere [Owen et al., 1977]. Throughout a Martian year, up to $30 \%$ of the total $\mathrm{CO}_{2}$ condenses onto the surface in winter polar regions [Kelly et al., 2006]. The condensation of $\mathrm{CO}_{2}$ occurs because the Martian atmosphere is sufficiently thin that transport of sensible heat is unable to sustain the winter polar atmosphere. As a result, the temperatures fall until the frost point of $\mathrm{CO}_{2}$ (also loosely referred to as "condensation point," although condensation usually refers to the phase change from gas to liquid) is reached, after which thermal infrared losses are buffered by latent heating.

[4] The Viking landers (VLs) provide the only interannual records of surface pressure on Mars. These records contain variability on several different time scales. Variations on time scales of seconds, hours, and days are associated with boundary layer turbulence, the thermal tides, and large-scale weather systems, for example. However, when the high frequency component is ignored, the surface pressure variation at a specific location on Mars (Figure 1) is largely determined by the bulk mass of atmosphere, though small contributions due to standing systems over long periods like the condensation flow and the intertropical lows and subtropical highs are also present. Therefore, to some significant degree of precision, the VL pressure records can be taken as topographically shifted records of the bulk atmospheric mass 

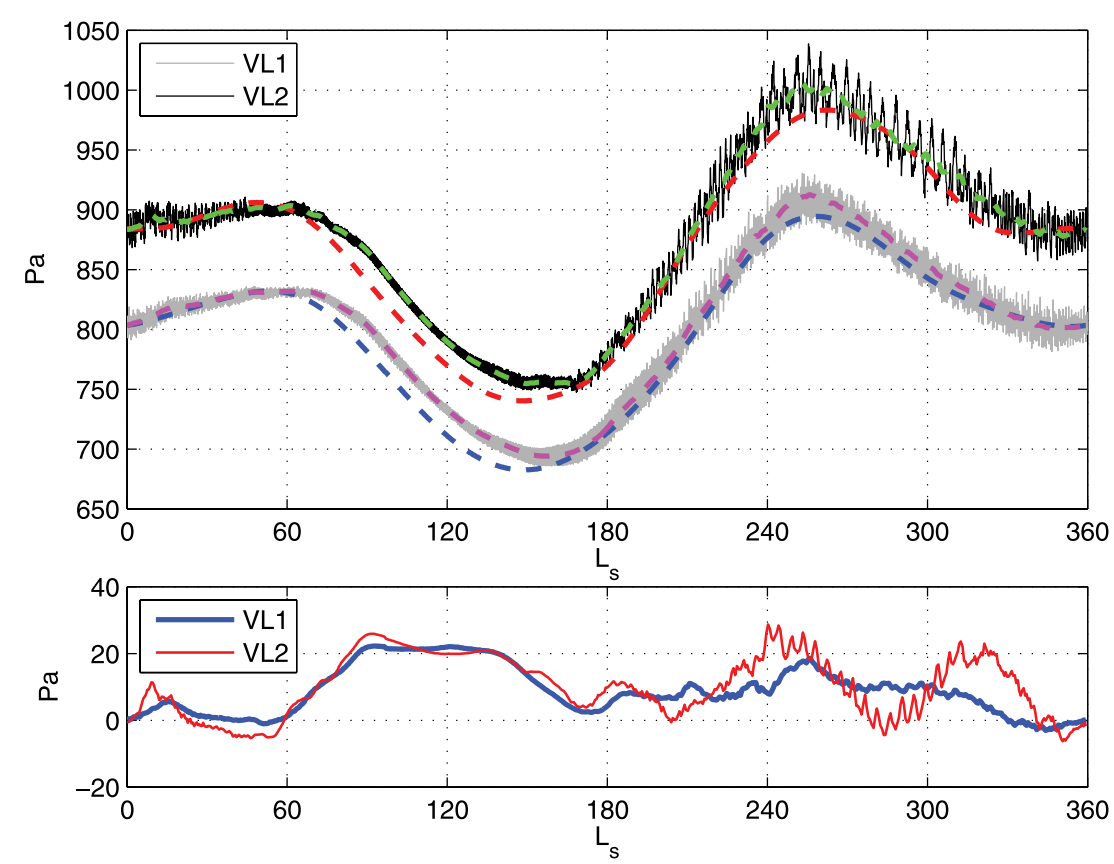

Figure 1. (top) Pressures at the VL sites for the control case (northern seasonal cap albedo, 0.77; northern seasonal cap emissivity, 0.57 ; south albedo, 0.5 ; south emissivity, 0.8 ; total $\mathrm{CO}_{2}$ mass index, 1.0 ). Grey line, MarsWRF surface pressure at VL1 location; magenta dashed line, smoothed MarsWRF surface pressure at VL1 location; black line, MarsWRF surface pressure at VL2 location; green dashed line, smoothed MarsWRF surface pressure at VL2 location; blue dashed line, smoothed VL1 observation; red dashed line, smoothed VL2 observation. (bottom) Difference between the smoothed VL measurements and MarsWRF simulation: blue line, VL1 site; red line, VL2 site.

[Hess et al., 1977]. Fitting the observed VL pressure cycle also establishes a baseline for climate studies, both short-term and long-term. As shown in Figure 1, the dominant longperiod signal is the very repetitive annual cycle that can be identified with the atmosphere freezing out to form the seasonal polar caps, followed by the caps' decay due to sublimation back to the atmosphere [Hourdin et al., 1993]. Models are often tuned to match this annual cycle, though small errors in amplitude and (especially) phase are often tolerated, depending on the interests of a particular study [Forget et al., 1998; Haberle et al., 2008; Hourdin et al., 1993; Pollack et al., 1993; Richardson and Wilson, 2002]. Indeed, the quality of fits in GCMs is in some sense surprisingly poor. For a system that should be driven primarily by radiative, latent, and thermal conductive heating processes at the caps, it would seem that a near perfect fit should be attainable. An accurate simulation of the pressure cycle would also be desirable for many applications where good prediction of the surface pressure is needed as a boundary condition, such as surface wind stress calculation, spacecraft entry-descent-landing analysis, etc. In reality, the main reason that ideal fits have not been attained is due to the computation involved: the problem reduces to one of searching for the best fit parameters using a relatively large number of simulations (certainly "large" by standards prior to the early 2000s).

[5] There are historical attempts to "fit" the $\mathrm{CO}_{2}$ cycle. Wood and Paige [1992] proved that it is possible to fit the VL data to within several Pascal using a one-dimensional (1-D) diurnal and seasonal thermal model without any explicit atmospheric contribution to the heat balance. The albedos and emissivities of the northern and southern seasonal $\mathrm{CO}_{2}$ caps and total mass of $\mathrm{CO}_{2}$ in the system were tuned and kept constant with time. In their 1-D study, the residual between the modeled pressure in the best case scenario and the observed was as small as a few Pascal. However, their best fit parameter values differ from the current estimates for the caps' radiative properties. If values from Wood and Paige [1992] are used in a GCM, the resulting pressure curves are not unreasonable, but the lack of an atmosphere and the use of constant thermal inertia translates to offsets that yields a GCM cycle notably worse than what the same parameters produces in the 1-D model.

[6] It is clear from 1-D modeling that tuning of the cap properties will allow the modeled $\mathrm{CO}_{2}$ cycle to be improved. It seems the standard for Mars GCMs is to accomplish this tuning "by-eye." However, the resulting parameters are not physical compared with spacecraft observations. For example, the cap albedos have to differ dramatically between the poles, with the northern values required to be much higher than observed [Haberle et al., 2008; Kieffer et al., 2000; Kieffer and Titus, 2001]. One possible way to improve the reasonableness of the parameters is to include other effects, such as the thermal conductivity induced by subsurface water ice [Haberle et al., 2008]. However, to date there has not been an attempt to systematically determine the cap properties needed in a GCM to produce a seasonal pressure cycle within the VL instrument error. Here, we present a recipe that can be used to calibrate a GCM pressure cycle, and we provide some discussion of what areas of uncertainty still remain in the understanding of the $\mathrm{CO}_{2}$ cycle on Mars. 
[7] In this paper, we describe the development and execution of a benchmark study for reproducing the observed surface pressure cycle in a Mars GCM. We specifically use the Martian implementation of the planetWRF model [Richardson et al., 2007]. The reason that Wood and Paige [1992] were able to generate excellent fits of the VL pressure cycle is that their 1-D model is simple and computationally cheap: hundreds of runs were made to cover the parameter space so that a best fit can be identified. Hundreds of runs for any sophisticated GCM is generally not feasible in a reasonable amount of time, nor are such runs necessary with our proposed method. Our technique involves the creation of an ensemble of simulations that are used to understand how the model's representation of the VL surface pressure cycles responds to perturbations of different parameters. Knowing these relationships, we use an iterative linear method to find the best set of parameters to fit the VL data. The method can fit the VL data with a very high degree of accuracy: comparable to, if not better than, the fit using the simpler onedimensional model [Wood and Paige, 1992]. This approach is non model specific and is suggested as an efficient means of finding the parameters for a best fit pressure cycle for any GCM. After demonstrating the method for the set of parameters chosen by Wood and Paige [1992], we proceed further to study how changes in soil thermal properties due to subsurface water ice effect the model's representation of the VL surface pressure record.

[8] The fact that Wood and Paige [1992] were able to fit the pressure cycle using a simple model has profound meaning. It suggests that the physics included in the model, namely the surface heat balance at the poles, is the controlling physics for modeling the $\mathrm{CO}_{2}$ and pressure cycles. The nonphysicality of the cap parameters retrieved from the Wood and Paige [1992] scheme does not invalidate the approach: instead it demonstrates that there exists a range of processes that effect the polar energy balance that are not incorporated ("resolved") in the model and hence their effects are "aliased" (or "bundled") into the estimated best fit parameters: for example, an unrealistic albedo value may effectively compensate for model-missing clouds. Further progress in understanding the $\mathrm{CO}_{2}$ cycle, which is tantamount to understanding the polar energy balance, is now a question of unbundling the various processes that are aliased into the retrieved parameters. The way this can be done is by applying models with more complete sets of explicit physics. Applying the same fitting routines within a hierarchy of increasingly complex models will allow the progressive unbundling of important physics affecting the polar heat balance. Where will it all end? At some point a set of physically plausible radiative parameters (potentially in the form of time and spatially varying parameters defined by a physical understanding of the ice microphysics and formation history) will signal the completion of the unbundling process. For example, in this study, we apply a GCM to the problem studied in 1-D by Wood and Paige [1992]. Explicit treatment of atmospheric dynamics, and spatially varying topography and regolith albedo and thermal properties can thus be unbundled from the parameters found by Wood and Paige [1992]. The complexity of implementing this task arrives from the very large numbers of runs required to find excellent data fits. Such a brute-force method is not available when running computationally expensive GCM simulations.
Hence, much of our discussion in this paper is in the demonstration of a method to allow data fitting with a relatively small number of runs. In principal, modified versions of this approach can be applied to fit various other data sets (e.g., temperature). In essence, it is a limited implementation of a prototype form of data assimilation (here we are pursing "parameter estimation" while most data assimilation attempts "state estimation"). Obviously, an ideal approach would be to simultaneously fit the surface pressure data along with records of air temperature, dust, water ice, etc. This in fact would require a full parameter and state data assimilation system, which is a future goal of our research. That notwithstanding, the approach and schemes presented here allow some new insight into the $\mathrm{CO}_{2}$ cycle without having to resort to a full data assimilation system.

[9] In section 2, we discuss the relevant physical processes contributing to surface energy balance, and we present our initial sensitivity study for several important contributing parameters. In section 3, we introduce the iterative linear fitting method. We show the fitting results in section 4. Physical interpretation of the best fit parameters is discussed in section 5. Section 6 concludes this paper.

\section{Surface Energy Balance and Sensitivity Study 2.1. Surface Energy Balance}

[10] The key physics for the Martian annual $\mathrm{CO}_{2}$ cycle is the surface energy balance. The instantaneous surface energy balance equation when $\mathrm{CO}_{2}$ frost is on the surface is

$$
S(1-a) \cos (i)-\varepsilon \sigma T^{4}+k d T / d z+L d m / d t=0,
$$

where $S$ is the incoming solar flux at the current Mars-Sun distance; $a$ is the albedo, either that of bare soil or frost; $i$ is the solar incidence angle; $\varepsilon$ is the surface emissivity, either that of bare soil or frost; $\sigma$ is the Stefan-Boltzmann constant for blackbody emission; $T$ is the surface temperature, either that of bare soil or the frost temperature when frost presents; $k$ is the thermal conductivity of the soil; $d T / d z$ is the vertical temperature gradient at the surface with $z$ positive downward (therefore, $k d T / d z$ is the upward conductive heat flux at the surface); $L$ is the latent heat of $\mathrm{CO}_{2}$ frost; $d m / d t$ is the $\mathrm{CO}_{2}$ frost deposition/sublimation rate. The thermal conductivity of a material is related to its thermal inertia $(I)$ by the equation $I^{2}=k \rho c$, where $\rho$ is the density and $c$ is the heat capacity [Wood and Paige, 1992]. The product of $\rho$ and $c$ is assumed to be $1.26 \times 10^{6} \mathrm{~J} \mathrm{~kg} \mathrm{~m}^{-6} \mathrm{~K}^{-1}$ in MarsWRF. This is a simplified view of the problem. The GCM contains additional terms associated with the atmospheric components that contribute to this energy balance, which are usually much smaller when compared to the rest. Thus for simplicity without losing clarity to the readers, we will refer to this equation, which captures the majority of the physics.

[11] At any given time and location, when the radiative and sensible heating terms in equation (1) are negative, latent heating is required to balance the cooling and maintain the temperature at the condensation point (the temperature remains at the condensation point because any infinitesimal temperature drop would yield a drop in the required saturation vapor pressure. The difference between the actual surface pressure and the infinitesimally lower saturation vapor pressure would drive $\mathrm{CO}_{2}$ from the atmosphere onto 
the surface, liberating latent heat that would tend to bring the temperature back up to the point where the atmospheric pressure and the saturation vapor pressure again agree). As a result, negative net radiative and sensible heating yields $\mathrm{CO}_{2}$ gas conversion to ice and deposition on the surface. When net heating is positive and $\mathrm{CO}_{2}$ ice is present on the surface, some amount of $\mathrm{CO}_{2}$ frost becomes gas until the surface is exhausted of all its $\mathrm{CO}_{2}$ ice cover. These phase exchange processes are usually assumed to be instantaneous in GCMs.

[12] Extensive work has been done examining water ice cloud and $\mathrm{CO}_{2}$ ice cloud formation. Modeling studies found evidence showing their importance in the polar energy budget by influencing the surface properties, temperature, and dynamics [Colaprete et al., 2005, 2008; Richardson et al., 2002]. Because the microphysics modeling of clouds in the Martian atmosphere is a subject under investigation, we do not include $\mathrm{CO}_{2}$ cloud formation or a water cycle in this study (these processes will await further unbundling at a future date).

[13] In selecting parameters to use for the fitting of the $\mathrm{CO}_{2}$ cycle, we take into account what variables are going to have a major effect on the polar energy balance and also what aspects of the model are relatively well constrained. For the radiative transfer, we use our standard solar and thermal infrared radiative heating schemes and also chose to prescribe the dust opacity distribution with the Mars Climate Database "MGS" scenario parameterization. Global soil albedo, emissivity and surface thermal inertia are used as derived from spacecraft observations [Richardson et al., 2007]. The parameters chosen to vary for the simulation are those associated with the seasonal caps and the bulk inventory of $\mathrm{CO}_{2}$ : the albedo and the emissivity of the seasonal $\mathrm{CO}_{2}$ ice caps (potentially separate values for each pole), the global $\mathrm{CO}_{2}$ mass (both gaseous and solid phase), and the thermal inertia of the polar regolith, which is a mixture of soil and water ice. All the parameters are crucial to the energy balance and the pressure cycle. In the sections following, we first discuss the conventional tuning parameters, namely albedo, emissivity, and total $\mathrm{CO}_{2}$ inventory. Then we discuss the less-studied varying thermal property of the subsurface.

\subsection{Usual Suspects: Albedo, Emissivity, and Total $\mathrm{CO}_{2}$ Inventory}

[14] The albedo determines the fraction of incoming solar energy reflected back to space. In general, increasing the albedo decreases the absorbed energy available to the landair system. In the Martian polar winter, as temperatures drop to the $\mathrm{CO}_{2}$ frost point and $\mathrm{CO}_{2}$ deposits on the surface, the high (bright) ice albedo further lowers solar heating, and more $\mathrm{CO}_{2}$ freezes out. The magnitude of this feedback effect depends on the value of the frost albedo. Thus, surface pressure tends to lower as a result of an increased $\mathrm{CO}_{2}$ cap albedo, and vice versa. We note that this effect is only relevant when $\mathrm{CO}_{2}$ frost is present and it is receiving insolation (an example of this is shown in Figure 3 (top), discussed later).

[15] The emissivity of the seasonal caps participates in maintaining surface energy balance differently than albedo does. The larger the emissivity of a $\mathrm{CO}_{2}$ frost cap, the more energy it releases to space. Thus a larger emissivity yields a larger energy deficit, which in turn requires more condensation of $\mathrm{CO}_{2}$ to release latent heat and compensate for the energy deficit. The net effect is that an increase in emissivity leads to a decrease in the surface pressure. Similarly to albedo's effect, we note that the effect of emissivity on surface pressure is only relevant when $\mathrm{CO}_{2}$ frost is present, though it can operate throughout polar night (this is also present in Figure 3 (top), discussed later). This means that the effects from albedo changes and from emissivity changes have different longevity and prominence: while albedo only acts when the seasonal caps are exposed to the sun, emissivity acts as long as the surface frost exists. Therefore, the albedo "footprint" (the effect on the surface pressure cycle due to variations of albedo) for a given pole is nonzero only later in its season of frost coverage, whereas the emissivity footprint is present throughout the frost coverage season.

[16] The total mass of $\mathrm{CO}_{2}$ in the system influences the surface pressure cycle in a very linear manner. Since none of the terms in equation (1) are very sensitive to total atmospheric mass, the surface frost amount changes very little when total mass of $\mathrm{CO}_{2}$ is changed (this is also present in Figure 3 (top), discussed later). Of course, one should not make very large perturbations to the $\mathrm{CO}_{2}$ inventory lest the planet migrate to another climate regime [Mischna et al., 2000]. It should also be noted that since the model does not predict the development of a residual $\mathrm{CO}_{2}$ ice cap (nor does any published GCM), increasing the surface pressure is possible by increasing the total $\mathrm{CO}_{2}$ inventory.

\subsection{Water Ice in the Subsurface Layer}

[17] In equation (1), the vertical heat flux is determined by the temperature gradient near the surface and the soil's thermal conductivity. Subsurface thermal structure is determined by the energy input from the surface and the thermal property underneath. Typically, the subsurface thermal conductivity is assumed to be the same as that of the surface. It has recently been suggested that the subsurface water ice would affect the exchange of $\mathrm{CO}_{2}$ between the atmosphere and the seasonal caps greatly [Haberle et al., 2008]. The presence of water ice increases the thermal conductivity of the soil. In the summer, when the surface is not covered by frost, more heat conducts downward compared to the situation where homogenous dry soil is usually assumed. When this extra amount of heat is released in the winter, less surface $\mathrm{CO}_{2}$ ice forms, and surface pressure increases accordingly. Therefore, changing the thermal inertia (equivalent to the thermal conductivity) of the regolith changes the thermal structure and heat flux of the soil. Eventually, it changes the surface pressure cycle.

[18] In order to investigate how subsurface water ice affects the $\mathrm{CO}_{2}$ cycle, we modified the subsurface model in the MarsWRF to handle vertical gradients of soil thermal conductivity. At depth where water ice becomes stable and has significant mass, we increase the thermal conductivity of the soil. This is similar to the two layer model used by Haberle et al. [2008], in which the regolith beneath a certain depth $(8.05 \mathrm{~cm}$ for the northern hemisphere and $11.16 \mathrm{~cm}$ for the southern hemisphere) was fully filled by a water ice table from $55^{\circ}$ to $90^{\circ}$ latitude (Figure 2, right); however, we do not use fixed depths across the two hemispheres.

[19] In order to parameterize the three-dimensional distribution of water ice in the subsurface, we include the water 

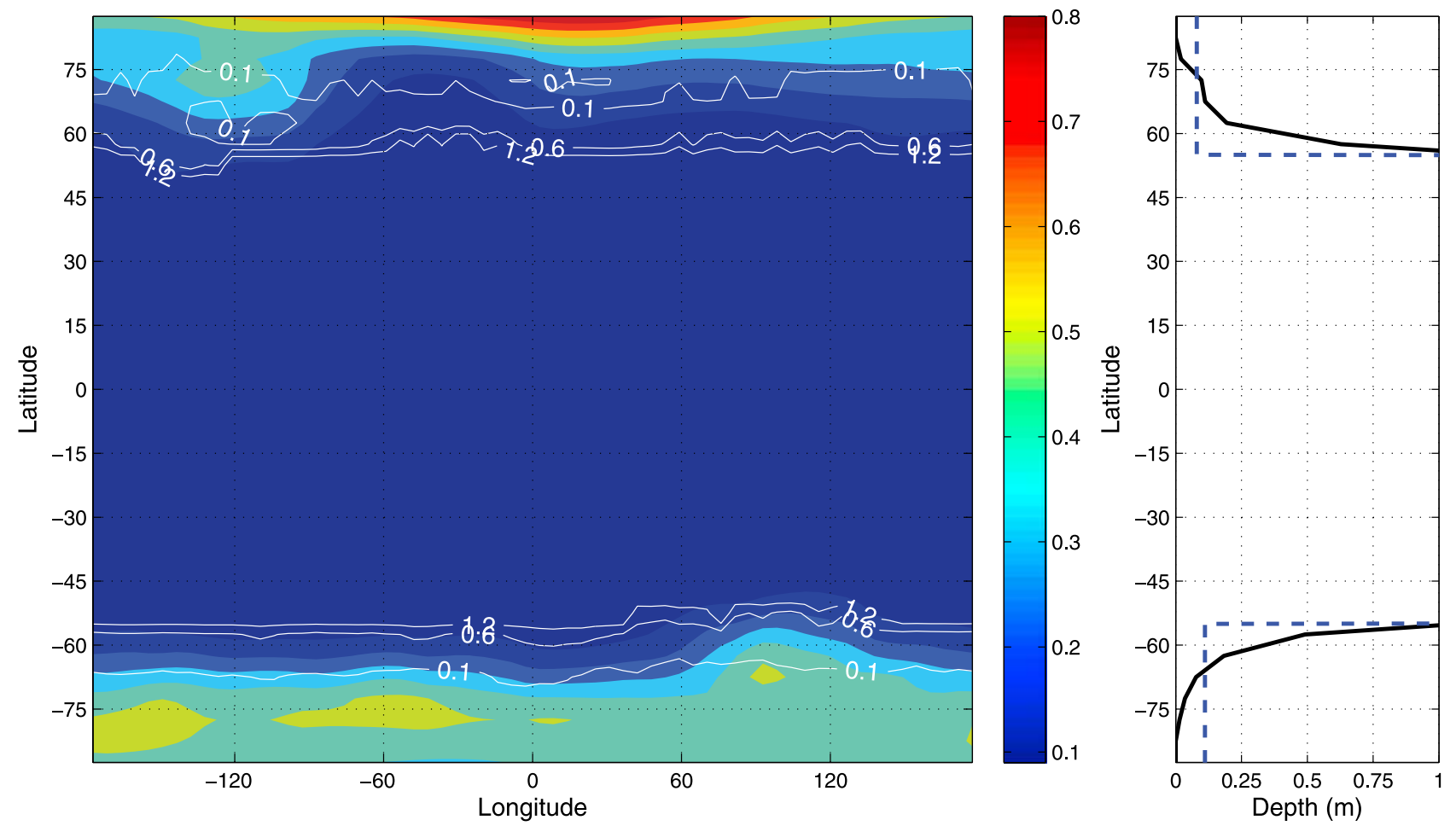

Figure 2. (left) Color shows the subsurface ice content from GRS; white contours indicate the depth of permanently stable water ice in meters suggested by Schorghofer and Aharonson [2005]. (right) Black curve indicates the zonal average of the depth of permanently stable water ice table; blue curve indicates the ice table depth used by Haberle et al. [2008].

ice content map provided by the Mars Odyssey Gamma Ray Spectrometer (GRS) [Boynton et al., 2002; Feldman et al., 2002, 2004] and the depth of the permanently stable subsurface water ice predicted by Schorghofer and Aharonson [2005] in MarsWRF (Figure 2). Water ice is only seasonally stable at midlatitudes [Schorghofer and Aharonson, 2005], and the available water vapor in the atmosphere does not seem to be enough to fill meters of regolith [Smith, 2002]. Therefore, we set the water ice table depth to be the cutoff line of the permanently stable water ice, which changes with latitude and longitude.

[20] The other parameter to decide upon is the thermal conductivity, or equivalently the thermal inertia, of the soil mixed with water ice (hereafter, we loosely call it a water ice table even though it is not pure water ice). Viking Infrared Thermal Mapper (IRTM) observations show that the apparent thermal inertia of the dry Martian surface ranges from 46 to $630 \mathrm{~J} \mathrm{~m}^{-3} \mathrm{~K}^{-1}$, with a global average of $275 \mathrm{~J} \mathrm{~m}^{-3} \mathrm{~K}^{-1}$ [Paige, 1992]. This agrees with observations from the Thermal Emission Spectrometer (TES) on board of Mars Global Surveyor (MGS) [Putzig et al., 2005]. Pure water ice has a thermal inertia of $2200 \mathrm{~J} \mathrm{~m}^{-3} \mathrm{~K}^{-1}$. Assuming a mixture has $80 \%$ of water ice, the thermal inertia of the mixture can range from 100 to $1200 \mathrm{~J} \mathrm{~m}^{-3} \mathrm{~K}^{-1}$, depending on the thermal inertia of the host regolith. These numbers provide physical constraints for the fitting parameters in later sections. We do not assume the water content provided by GRS for the water ice table because that data set is not vertically well resolved. The water information from GRS is only used for the geographical distribution. We set the threshold for water content to $9 \%$ in order to cover the latitudes of $55^{\circ}$ and poleward.

[21] Of course, we do not have to limit ourselves to these numbers in the sensitivity study. We find that the VL pressure cycle is very sensitive to the thermal inertia assumed for the water ice table (Figure 3, bottom). A change in the assumed water ice table thermal inertia changes the VL pressure cycle significantly. As expected, larger thermal inertia leads to higher surface pressure. We find that the water ice table thermal inertia shows a very similar footprint to that of the emissivity: the increasing phase of their signals lasts from Ls $210^{\circ}$ to $360^{\circ}$ in the northern winter and from Ls $30^{\circ}$ up to $180^{\circ}$ in the southern winter; the decreasing phase of the thermal inertia signal ends shortly before that of the emissivity. However, we find that thermal inertia does not project a change on the surface pressure cycle as linearly as emissivity does. For example, doubling the thermal inertia perturbation does not necessarily double the resulting pressure change. In addition, changing the thermal inertia modifies the phase of the pressure signal. Also of note, the sensitivity to thermal inertia evidently saturates: when we extend the thermal inertia above a certain amount, the pressure cycle fails to respond. At that point, the soil in the model contains the maximum possible amount of heat it can. Fortunately for the prospect of fitting the pressure cycle, with the current depth of soil, MarsWRF seems to reach that extreme for a thermal inertia value larger than $5000 \mathrm{~J} \mathrm{~m}^{-3} \mathrm{~K}^{-1}$, which is much larger than most of the possible material in the regolith including pure water ice. Nonetheless, we need to be careful when we perform the linear retrieval later, where we assume 

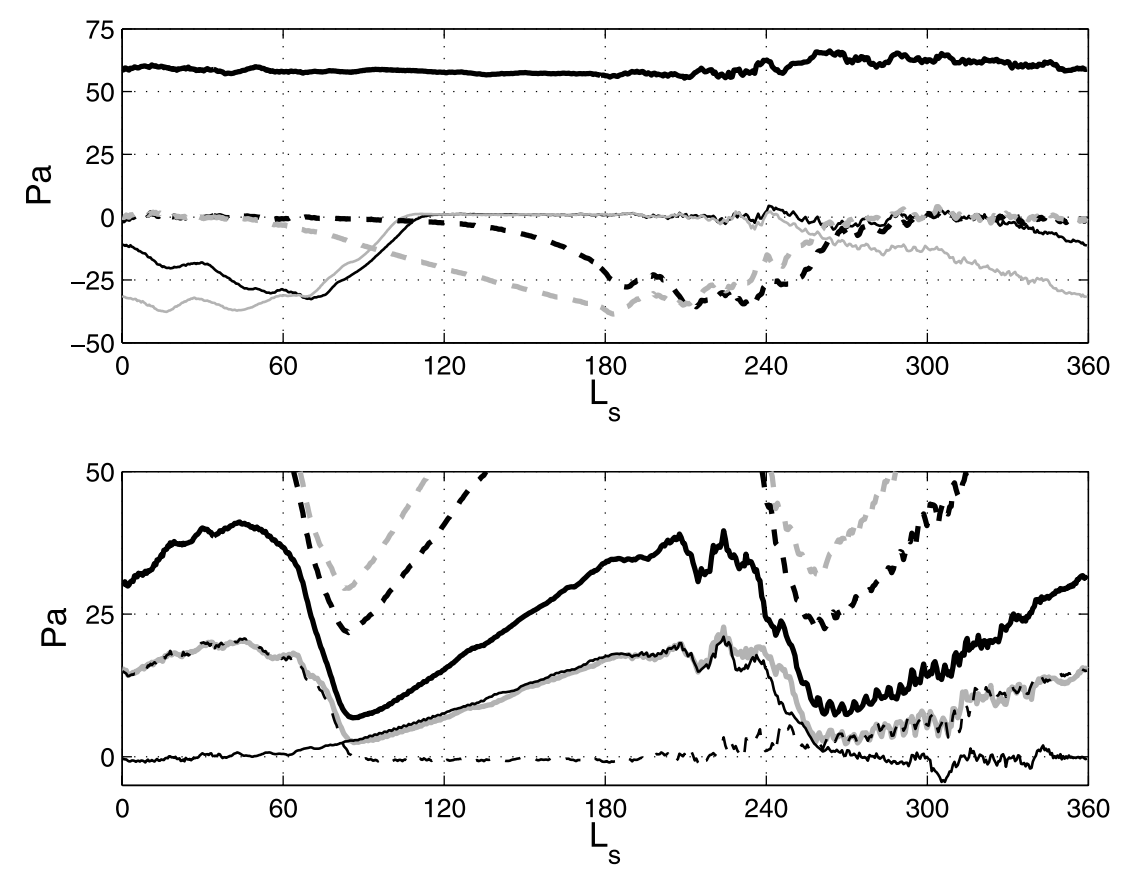

Figure 3. Response of MarsWRF surface pressure at VL1 location after positive perturbations. (top) Perturbation to north seasonal cap albedo (thin black line, $\boldsymbol{P}_{1}$ ), north emissivity (thin gray line, $\boldsymbol{P}_{2}$ ), south albedo (thick black dash line, $\boldsymbol{P}_{3}$ ), south emissivity (thick gray dash line, $\boldsymbol{P}_{4}$ ), and total $\mathrm{CO}_{2}$ mass (thick black line, $\boldsymbol{P}_{5}$ ). (bottom) Perturbation to the thermal inertia of the water ice table at the north polar region by $600 \mathrm{~J} \mathrm{~m}^{-3} \mathrm{~K}^{-1}$ (thin black dash line, $\boldsymbol{P}_{6}^{e}$ ), south by $600 \mathrm{~J} \mathrm{~m}^{-3} \mathrm{~K}^{-1}$ (black line, $\boldsymbol{P}_{7}^{e}$ ), both by $600 \mathrm{~J} \mathrm{~m}^{-3} \mathrm{~K}^{-1}$ (thick gray line, $\boldsymbol{P}_{\text {extra }}^{e}$ ), both by $1000 \mathrm{~J} \mathrm{~m}^{-3} \mathrm{~K}^{-1}$ (thick black line), both by $2000 \mathrm{~J} \mathrm{~m}^{-3} \mathrm{~K}^{-1}$ (thick black dash line), and both by $3000 \mathrm{~J} \mathrm{~m}^{-3} \mathrm{~K}^{-1}$ (thick gray dash line). The last two curves only appear partially because of the scale limit.

the pressure cycle always responses linearly to all the perturbations.

[22] The average thermal inertia of dry Martian soil has an annual skin depth around $1 \mathrm{~m}$, which is usually deeper than the stable water ice depth. Our subsurface model in MarsWRF covers more than $7 \mathrm{~m}$, deeper than the annual skin depth of pure water ice (thermal inertia of $2200 \mathrm{~J} \mathrm{~m}^{-3} \mathrm{~K}^{-1}$, skin depth $5 \mathrm{~m}$ ). The stable water ice depth was calculated assuming the soil porosity is about $70 \%$. The pressure cycle is not very sensitive to the assumption of the underlying soil porosity. This is because the stable ice depth does not change much with soil porosity near the poles, where the low temperature allows subsurface water ice to preserve easily.

\subsection{Mathematical Representation}

[23] In order to simplify the discussion and the description of the linear fitting method, we introduce the following mathematical notation. In the initial experiments, five parameters in MarsWRF are tuned: the albedo and emissivity of the seasonal $\mathrm{CO}_{2}$ caps for both the north and south poles, and the total mass of $\mathrm{CO}_{2}$ in the system. The linear methodology discussed in section 3 relies on making relatively small corrections; hence we constructed a baseline case with parameters similar to those used by Wood and Paige [1992]. The northern cap albedo is set to 0.770, northern cap emissivity to 0.570 , southern cap albedo to 0.500 , southern cap emissivity to 0.800 and total $\mathrm{CO}_{2}$ mass to
$2.90 \times 10^{16} \mathrm{~kg}$ of $\mathrm{CO}_{2}$ (we use an index number 1.00 hereafter to represent this reference total $\mathrm{CO}_{2}$ amount). We denote the parameter vector as $\boldsymbol{A}$, a column vector whose rows contain the parameters used in an experiment in the order just mentioned. For the baseline case, we write

$$
\boldsymbol{A}_{0}=\left[\begin{array}{lllll}
0.770 & 0.570 & 0.500 & 0.800 & 1.00
\end{array}\right]^{\mathrm{T}},
$$

where superscript $\mathrm{T}$ denotes vector transpose. The values in this parameter vector are used in MarsWRF to yield its corresponding baseline model atmosphere. After the model reaches a steady state (taken to be a Martian year; note that the initial subsurface temperature were taken from the results of a prior decadal simulation and were thus very nearly in balance from the outset), a Mars year of surface pressure output is diagnosed for its predicted values of the Viking Lander 1 (VL1) data record. This diagnosis requires interpolating within the output to find the surface pressure at the actual latitude, longitude, and elevation of the lander (gray line in Figure 1). It is evident that the model's predicted pressure cycle has larger short-term variations in the second half of the year, and this is consistent with observations and previous GCM studies. Details of those short-term variations are beyond the scope of this study. For the purpose of tuning the uncertain parameters in our vector, we are only interested in the long-term trend. Hence we apply a 9-day running averaging to the model output's predicted surface pressure 
record to remove the undesired high frequency components. This smoothed pressure cycle, $\boldsymbol{X}_{0}$, a column vector with 669 rows (each row represents a sol) is plotted in Figure 1 as the dashed magenta line. We write

$$
\boldsymbol{X}_{0}=I\left(W\left(\mathbf{A}_{0}\right)\right) \equiv F\left(\boldsymbol{A}_{0}\right)
$$

where operator $I$ denotes all the required interpolations and smoothing, and operator $W$ denotes the MarsWRF model when run with the parameters included in $\boldsymbol{A}_{0}$. We can combine these two operators into one effective operator, $F$, that maps a given parameter vector to a predicted smoothed VL1 pressure record.

[24] In order to sensibly compare our smoothed model predicted pressure cycle to the observations, which also exhibit high frequency variations due to tides and baroclinic weather systems, we have used a smoothed, continuous representation of the VL1 observations in non dust storm conditions. Instead of applying the same 9-day running average as we have to the model output, we use the polynomial fit of the non dust storm VL1 pressure curve [Tillman et al., 1993]. Using a polynomial allows us to compare the model to the data in regions where there are gaps in the VL1 data record. The smoothed VL1 observations are presented by the dashed blue line in Figure 1, with one value of VL1 surface pressure per sol (669 values in a Martian year). We follow the same practice with the VL2 observation, shown in Figure 1 as the red dashed curve, though we do not use the VL2 observations within the linear fitting method.

[25] As is evident in Figure 1, the smoothed pressure cycle generated using the baseline parameter set (magenta dashed line for VL1 and green dashed line for VL2) differs from the observations (blue dashed line for VL1 and red dashed line for VL2). The largest differences are in the northern summer (or the southern winter), where phase errors are evident. The residual, defined as the model predictions less the VL observations, vary from 0 to $20 \mathrm{~Pa}$ throughout most of the year, with an average of $8.2 \mathrm{~Pa}$ and a standard deviation of $7.0 \mathrm{~Pa}$ at the VL1 site. The root mean square (RMS) of the residual at the VL1 site is $10.8 \mathrm{~Pa}$, or $1.2-1.6 \%$ of the seasonal cycle.

[26] To estimate the sensitivities of MarsWRF to the parameters within $\boldsymbol{A}_{0}$, we make five additional MarsWRF model runs, one for each element in $\boldsymbol{A}_{0}$. Each run adds a small perturbation to the value of one parameter in $\boldsymbol{A}_{0}$ while keeping the other values unchanged. Once completed, we apply the same interpolation and averaging to obtain the five predicted smooth pressure cycles at the VL1 location associated with the five perturbed parameter vectors. We denote the perturbed parameter vectors as $\boldsymbol{A}_{i}(i=1,2,3,4,5)$, and analogously to equation (3), their resulting pressure records are $\boldsymbol{X}_{i}(i=1,2,3,4,5)$. To be clear, the order of the perturbations associated with the $i$ index follows the order of parameters within $\boldsymbol{A}_{0}$ : northern seasonal cap albedo, northern cap emissivity, southern cap albedo, southern cap emissivity and the index number of the total $\mathrm{CO}_{2}$ mass in MarsWRF, respectively. We chose the following set of perturbed parameter vectors $\boldsymbol{A}_{i}$ :

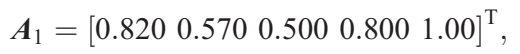

$$
\begin{aligned}
& \boldsymbol{A}_{2}=\left[\begin{array}{lllll}
0.770 & 0.670 & 0.500 & 0.800 & 1.00
\end{array}\right]^{\mathrm{T}}, \\
& \boldsymbol{A}_{3}=\left[\begin{array}{lllll}
0.770 & 0.570 & 0.600 & 0.800 & 1.00
\end{array}\right]^{\mathrm{T}},
\end{aligned}
$$

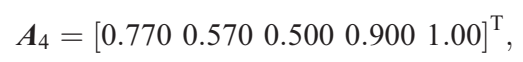

$$
\boldsymbol{A}_{5}=\left[\begin{array}{lllll}
0.770 & 0.570 & 0.500 & 0.800 & 1.06
\end{array}\right]^{\mathrm{T}} \text {. }
$$

These vectors are related to the baseline parameter vector through

$$
\boldsymbol{D}_{i}=\boldsymbol{A}_{i}-\boldsymbol{A}_{0},(i=1,2,3,4,5)
$$

Similarly, we define the perturbation pressure vectors

$$
\boldsymbol{P}_{i}=\boldsymbol{X}_{i}-\boldsymbol{X}_{0}=F\left(\boldsymbol{A}_{i}\right)-F\left(\boldsymbol{A}_{0}\right),(i=1,2,3,4,5) .
$$

While a matrix whose columns are the $\boldsymbol{D}_{i}$ vectors is a $5 \times 5$ diagonal matrix, a matrix whose columns are the $\boldsymbol{P}_{i}$ vectors is $669 \times 5$ and potentially has nonzero entries everywhere. The $\boldsymbol{P}_{i}$ vectors are shown in Figure 3 (top), and one can see that they are in general nonzero, except for the vectors associated with perturbed albedo and emissivity when they are not contributing to the surface energy balance.

[27] This same procedure for gauging the model's sensitivity to uncertain parameters can easily incorporate other components. Later in this study, we extend the parameter vector to include the water ice table thermal inertia. This requires defining a new baseline case with parameter vector

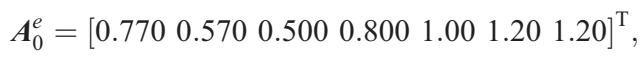

where the definitions of the first five elements of this vector are the same as before, and the sixth and seventh elements correspond to the thermal inertia of the subsurface layer in the northern polar region and the southern polar region, in units of $1000 \mathrm{~J} \mathrm{~m}^{-3} \mathrm{~K}^{-1}$. We use the same notation for the operators that map a given parameter vector to a predicted smoothed pressure curve for VL1:

$$
\boldsymbol{X}_{0}^{e}=I\left(W\left(\boldsymbol{A}_{0}^{e}\right)\right)=F\left(\boldsymbol{A}_{0}^{e}\right)
$$

To be clear, in the experiments where only the first five parameters are varied, MarsWRF still runs with assumed values for the sixth and seventh parameters. However, their values are not available for modifying to better fit the VL1 pressure data. When the parameters for subsurface thermal inertia in the two hemispheres are available for change, we specify two new parameter vectors:

$$
\begin{aligned}
& \boldsymbol{A}_{6}^{e}=\left[\begin{array}{lllllll}
0.770 & 0.570 & 0.500 & 0.800 & 1.00 & 1.50 & 1.20
\end{array}\right]^{\mathrm{T}}, \\
& \boldsymbol{A}_{7}^{e}=\left[\begin{array}{lllllll}
0.770 & 0.570 & 0.500 & 0.800 & 1.00 & 1.20 & 1.50
\end{array}\right]^{\mathrm{T}} .
\end{aligned}
$$

These extended perturbed parameter vectors logically extend to the definitions of $\boldsymbol{D}_{6}^{e}, \boldsymbol{D}_{7}^{e}, \boldsymbol{P}_{6}^{e}$ and $\boldsymbol{P}_{7}^{e}$ via equations (9) and (10). The corresponding perturbation pressure vectors for $\boldsymbol{P}_{6}^{e}$ 
and $\boldsymbol{P}_{7}^{e}$ are shown in Figure 3 (bottom). Also shows is one additional perturbation pressure vector obtained from running MarsWRF with

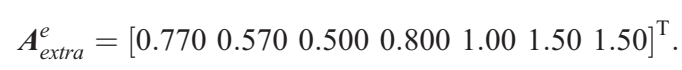

This is included to demonstrate that $\boldsymbol{P}_{\text {extra }}^{e}$ is roughly the sum of $\boldsymbol{P}_{6}^{e}$ and $\boldsymbol{P}_{7}^{e}$, thereby demonstrating these parameters behave fairly linearly. Note that $\boldsymbol{P}_{\text {extra }}^{e}$ is not used in the fitting method described in section 3 .

\section{Linear Fitting Methods}

[28] We chose to fit the pressure cycle at just one of the VL sites, reserving the other as an independent resource to check the retrieved set of parameters. Since the VL1 pressure record is much less susceptible to local weather disturbances and large global dust storms, and is more complete in turns of time coverage than the VL2 record, the VL1 data are taken to be the preferable target for model fitting, as was usually used in earlier studies [Forget et al., 1998; Wood and Paige, 1992]. It should be noted, however, that the method we employ can easily include VL2 data simultaneously.

[29] The Viking pressure transducers were calibrated to about $2 \mathrm{~Pa}$ [Tillman, 1988]. This $2 \mathrm{~Pa}$ instrument error is the only meaningful number that one can ascribe to "good enough" in regards to fitting the observations. To fit better than $2 \mathrm{~Pa}$ could be unwarranted and to fit worse than $2 \mathrm{~Pa}$ betrays shortcomings in the model. Without some reference to a meaningful numerical value, all fits are more or less qualitative.

[30] In order to fit the smoothed VL1 pressure cycle, we use a simple linear method to first construct a best fit pressure curve from the baseline scenario plus a linear combination of the 5 perturbation pressure vectors $\boldsymbol{P}_{i}$. We then assume, as a consequence of linearity, the resulting best fit MarsWRF parameters are equal to the baseline parameters plus the same linear combination of the perturbation parameter vectors, $\boldsymbol{D}_{i}$. If the linearity assumption holds, then we should find that nonlinearly validating the best fit parameters (by running MarsWRF with those values and applying the interpolation and smoothing operators) yields a pressure cycle very similar to both the best fit pressure curve found from the linear combination of $\boldsymbol{P}_{i}$, and the smoothed VL1 measurements.

[31] To illustrate the assumptions and consequences of our linearity assumption, consider a vector of perturbations, $\delta \boldsymbol{A}$, to the baseline parameter vector. If the nonlinear operator $F$ is applied to this perturbed parameter vector, then

$$
\boldsymbol{X}_{0}+\delta \boldsymbol{X}=F\left(\boldsymbol{A}_{0}+\delta \boldsymbol{A}\right)
$$

Performing a Taylor series expansion of the right hand side of equation (15) about the baseline vector:

$$
\boldsymbol{X}_{0}+\delta \boldsymbol{X}=F\left(\boldsymbol{A}_{0}\right)+\mathbf{F} \delta \boldsymbol{A}+\mathrm{O}\left(\|\delta \boldsymbol{A}\|^{2}\right)
$$

where $\mathbf{F}$ is the Jacobian matrix of partial derivatives of $F\left(\boldsymbol{A}_{0}\right)$ with respect to the elements in $\boldsymbol{A}$, evaluated about $\boldsymbol{A}_{0}$. Note that in our case $\mathbf{F}$ is a $669 \times 5$ matrix. If the vector of parameter perturbations is small, where "small" is defined by the ratio of the vector norms of $\delta \boldsymbol{A}$ and $\boldsymbol{A}_{0}$ being less than 1 , then one can safely neglect the higher-order terms in equation (16), represented by $\mathrm{O}\left(\|\delta \boldsymbol{A}\|^{2}\right)$. Neglecting these terms and subtracting the relation in equation (3) yields

$$
\delta \boldsymbol{X}=\mathbf{F} \delta \boldsymbol{A}
$$

which is a linear relationship between perturbations in parameters and their resulting perturbations in the pressure curve. An immediate consequence of this linear relationship is that a constant factor change in $\delta \boldsymbol{A}$ gives the same factor change in $\delta \boldsymbol{X}$.

[32] The potential complexity of equation (17) is contained within the matrix $\mathbf{F}$ (which itself is defined in equations (3) and (16)). While it is theoretically possible to evaluate the Jacobian matrices of MarsWRF $(W)$ with respect to specific parameters (i.e., the derivative of the model variables with respect to the parameters) and also for the interpolation and smoothing operators $(I)$ for MarsWRF output, it is a difficult feat requiring lots of code development. Instead of attempting to explicitly calculate $\mathbf{F}$, we approximate it by way of our explicit introduction of small perturbations to the parameters within $\boldsymbol{A}_{O}$ (as described in section 2). Hence, the columns of $\mathbf{F}$ are related to the perturbation pressure vectors and the magnitudes of parameter perturbations:

$$
\boldsymbol{F}_{i}=\boldsymbol{P}_{i} /\left\|\boldsymbol{D}_{i}\right\|,(i=1,2,3,4,5),
$$

where $\boldsymbol{F}_{i}$ are the columns of the $\mathbf{F}$ matrix. Alternatively, since the matrix with $\boldsymbol{D}_{i}$ as its columns, which we denote $\mathbf{D}$, is diagonal, then $\mathbf{F}=\mathbf{P D}^{-1}$, where $\mathbf{P}$ is the perturbation matrix with $\boldsymbol{P}_{i}$ as its columns and the superscript " -1 " denotes the matrix inverse.

[33] To fit the smoothed VL1 data, we assume

$$
\boldsymbol{Y}=\boldsymbol{X}_{0}+\mathbf{P} \boldsymbol{\alpha}+\varepsilon
$$

where $\boldsymbol{Y}$ denotes the smoothed VL1 data, $\boldsymbol{\alpha}$ is a column vector $(5 \times 1)$ of linear combination coefficients, and $\varepsilon$ is a column vector $(669 \times 1)$ of residuals. We find the best fit linear combination coefficient via an iterative method that minimizes the L2 norm (the root mean square) of $\varepsilon$. Hence, we define a quadratic cost function that gauges the (possibly weighted) magnitude of the residual vector:

$$
C(\boldsymbol{\alpha})=0.5 \times \varepsilon^{\mathrm{T}} \mathbf{W} \boldsymbol{\varepsilon}=0.5 \times\left(\boldsymbol{Y}-\boldsymbol{X}_{0}-\mathbf{P} \boldsymbol{\alpha}\right)^{\mathrm{T}} \mathbf{W}\left(\boldsymbol{Y}-\boldsymbol{X}_{0}-\mathbf{P} \boldsymbol{\alpha}\right),
$$

where $\mathbf{W}$ is a diagonal matrix that can be used to assign uneven weights to elements of the noise vector at different times of the year (if desired). The cost function in equation (20) is of a standard form and can be easily minimized by many different algorithms; because $\boldsymbol{\alpha}$ has only 5 or 7 elements here, we employ a straightforward downhill simplex method. When the minimizing algorithm reaches a (possibly local) minimum for the cost function $C$, the corresponding $\boldsymbol{\alpha}=\boldsymbol{\alpha}_{f}$ minimizes $\varepsilon$. The obtained best fit for $\boldsymbol{Y}$ based on the linear combination is then

$$
\boldsymbol{X}_{f}=\boldsymbol{X}_{0}+\mathbf{P} \boldsymbol{\alpha}_{f}
$$


[34] As the ultimate goal of this method is to find the MarsWRF parameter set that best fits the smoothed VL1 record, we need to relate $\boldsymbol{X}_{f}$ in equation (21) to $\boldsymbol{A}_{f}$, the best fit parameter set. If the linear truncation of the Taylor series in equation (16) holds, then changes in $\boldsymbol{X}$ can be linearly related to changes in $\boldsymbol{A}$, as in equation (17). Hence, we assume that the best fit parameter vector is related to the baseline parameter vector by the same linear combination coefficients that relate the best fit pressure cycle to the baseline pressure cycle prediction:

$$
A_{f}=A_{0}+\mathbf{D} \alpha_{f} .
$$

In order to gauge the success of the fitting method, and thus the validity of the linearity assumption, one must run MarsWRF with parameters $\boldsymbol{A}_{f}$ and apply the interpolation and smoothing operator to the output:

$$
\boldsymbol{X}_{f}^{\prime}=\mathrm{F}\left(\boldsymbol{A}_{f}\right)
$$

[35] If $\boldsymbol{X}_{f}^{\prime}$ is very similar to the linear method prediction, $\boldsymbol{X}_{f}$, then we can be assured the linearity assumption was valid. If $\boldsymbol{X}_{f}^{\prime}$ and $\boldsymbol{X}_{f}$ differ substantially, then one must reevaluate use of this method, though we have found that for poorly chosen baseline parameters, successive applications of the method can eventually yield an acceptable solution for $\boldsymbol{A}_{f}$.

[36] When we write $\boldsymbol{X}=\mathrm{F}(\boldsymbol{A})$, we consider the surface pressure to be a function of the albedo and the emissivity of the polar caps and the total mass of $\mathrm{CO}_{2}$ in the system (the extended vector also considers the thermal inertia of the subsurface ice table). By virtue of the linearity assumption, this method effectively retrieves these parameters $\boldsymbol{A}_{f}$, from observations of surface pressure, even though the involved operators $(I$ and $W)$ are nonlinear. In this respect, this linear fitting method is not new. The method is essentially a standard practice in atmospheric spectroscopy data retrieval [e.g., Guo et al., 2007] and data assimilation [e.g., Menemenlis et al., 2005].

\section{Results}

\subsection{Standard Fitting With Emissivity, Albedo,} and Total $\mathrm{CO}_{2}$ Mass

[37] We obtain a positive perturbation matrix, $\mathbf{P}^{+}$, to replace $\mathbf{P}$ in equation (19):

$$
\mathbf{P}^{+}=\left[\begin{array}{lllll}
\boldsymbol{P}_{1} & \boldsymbol{P}_{2} & \boldsymbol{P}_{3} & \boldsymbol{P}_{4} & \boldsymbol{P}_{5}
\end{array}\right] .
$$

We describe it as "positive" because all the columns in $\mathbf{P}^{+}$ were obtained by setting one of the five parameters larger than that in the baseline case and differencing the resulting pressure cycles. Similarly, we can construct a negative perturbation matrix $\mathbf{P}^{-}$, based on negative perturbations. We fit the VL1 pressure record with these two perturbation matrices and obtain two best fit parameter vectors (also listed in Table 1):

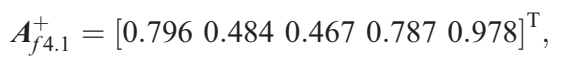

$$
\begin{aligned}
& \boldsymbol{A}_{f 4.1}^{-}=\left[\begin{array}{lllll}
0.793 & 0.485 & 0.454 & 0.784 & 0.978
\end{array}\right]^{\mathrm{T}} .
\end{aligned}
$$

The values in these two parameter vectors are very close. We average the two best fit parameter sets to get the average best fit parameter vector:

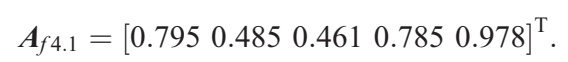

The two linear fits corresponding to $\boldsymbol{A}_{f 4.1}^{+}$and $\boldsymbol{A}_{f 4.1}^{-}$, and their average are shown in Figure 4 (top); their residuals are shown in Figure 4 (bottom). All fitted curves match very closely with the smoothed VL1 data. The largest misfits are found in the northern winter, when baroclinic waves are most active [Hess et al., 1977]. The largest error is less than $10 \mathrm{~Pa}$, or about $1 \%$ of the annual maxima.

[38] We use the average parameter vector $\boldsymbol{A}_{f 4.1}$ to drive MarsWRF. Figure 5 shows the resulting model output. The pressure cycles corrected for VL positions are indicated by the black and the gray curves. The smoothed model surface pressure cycles (green and magenta dashed curves) show great agreements with the smoothed VL data (red and blue dashed curves) at both landing sites (note that only the VL1 data were used for the fit). Figure 5 (bottom) shows the difference between the smoothed simulation and the smoothed data. For the VL1 site, the error is always less than several Pascal. The residual mean is $0.3 \mathrm{~Pa}$ and the standard deviation is $3.2 \mathrm{~Pa}$. The RMS of the residual is $3.2 \mathrm{~Pa}$, or $0.35-0.48 \%$ of the seasonal cycle. MarsWRF predicts slightly higher surface pressure near Ls $=20^{\circ}$ and $240^{\circ}$, lower surface pressure near Ls $=150^{\circ}$ and $270^{\circ}$. Similar, if not identical, residual patterns can be found in the linear fit (blue dashed line in Figure 4, bottom). It suggests that our final perturbation to $\boldsymbol{A}_{0}$ is small enough for linearity to hold (equation (19)). In this regime, the operator F can be considered close to linear. The translation back to the parameter space is therefore valid and reflected directly in the forward model (i.e., MarsWRF) output.

[39] We notice that the smoothed surface pressure cycle at the VL2 site follows the observation closely in most of the year, but it does not line up perfectly with the observations in the northern winter and around $\mathrm{Ls}=50^{\circ}$. Because we do not perform the pressure fitting for the VL2 data, such discrepancy is not surprising. Nonetheless, the fact that the model agrees with data even at the VL2 site for the majority of the year is impressive. It suggests that the hydrostatic assumption and the dynamics in MarsWRF are consistent with what actually happens on Mars. If pressure records at both VL sites are to be fit simultaneously, we would need to include the VL2 pressure responses in the perturbation matrix and in the definition of the cost function. A tradeoff of accuracy between the two landing locations is therefore expectable. For the reasons discussed at the beginning of section 3 , we decided to perform the fitting only for the VL1 pressure cycle.

[40] If achieving a better fit for a desired time frame is the goal, we can adjust the weighting assigned to different periods of time by modifying matrix $\mathbf{W}$ in the definition of the cost function (equation (20)). In Figures 4 and 5, one can find a subtle phase error at the pressure minima near Ls $=$ $150^{\circ}$. In order to improve the fitting quality at this season, we weight this period relatively more in the calculation of the cost function. We chose to assign 50 times the normal weighting to southern winter $\left(\mathrm{Ls}=125^{\circ}\right.$ to $\left.175^{\circ}\right), 10$ times 

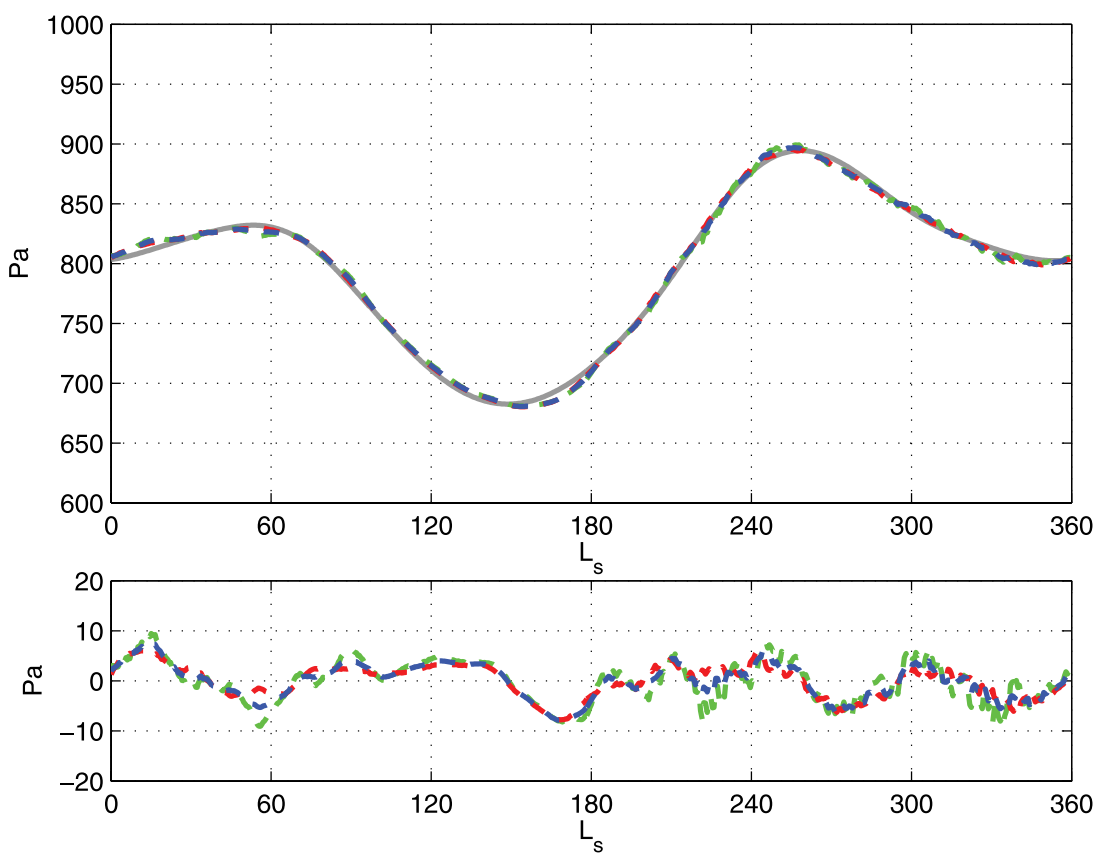

Figure 4. The linear fit of surface pressure cycle at VL1 site. (top) Gray line, smoothed VL1 data; green dashed line, linear fit result using positive perturbation matrix $\mathbf{P}^{+}$; red dashed line, linear fit result using negative perturbation matrix $\mathbf{P}^{-}$; blue dashed line, the average of the previous two linear fits. (bottom) Green dashed line; residual in the fit using $\mathbf{P}^{+}$; red dashed line, residual in the fit using $\mathbf{P}^{-}$; blue dashed line, the average residual.

to southern summer ( $\mathrm{Ls}=225^{\circ}$ to $275^{\circ}$ ) and normal weighting at all other times. With the updated weight matrix, the following best fit parameter vectors are given by the linear fitting method (also shown in Table 1):

$$
\begin{aligned}
& \boldsymbol{A}_{f 4.2}^{+}=\left[\begin{array}{lllll}
0.814 & 0.424 & 0.461 & 0.744 & 0.966
\end{array}\right]^{\mathrm{T}}, \\
& \boldsymbol{A}_{f 4.2}^{-}=\left[\begin{array}{lllll}
0.820 & 0.434 & 0.433 & 0.764 & 0.971
\end{array}\right]^{\mathrm{T}} .
\end{aligned}
$$

We average the two to obtain

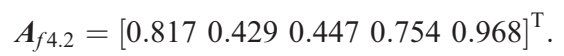

This parameter vector provides an excellent linear fit as well, with residual mean of $0.5 \mathrm{~Pa}$ and standard deviation of 4.6 Pa. When validated using MarsWRF, the RMS of the residual is $4.8 \mathrm{~Pa}$, or $0.53-0.70 \%$ of the pressure level. In both linear fit and model output, the residual in the southern winter $\left(\mathrm{Ls}=125^{\circ}\right.$ to $\left.175^{\circ}\right)$ is smaller and the phase shift is less evident. The trade off, however, is that the errors at other seasons become larger, which leads to a larger total RMS error.

[41] The experiments above suggest that the southern seasonal cap has low albedo and high emissivity, while the northern seasonal cap has high albedo and low emissivity. There is no intuitive explanation for why the two seasonal $\mathrm{CO}_{2}$ caps would have opposite radiative properties. Observation from TES and Viking IRTM do not support such a dichotomy in general [Kieffer et al., 1977; Kieffer and Titus, 2001; Paige et al., 1994; Paige and Keegan, 1994]. With just the fitting parameters used in this section, the southern parameters seem to match the observation from spacecrafts much better than those retrieved for the north.

\subsection{Fitting With Extended Parameterization of Subsurface Water Ice}

[42] Since the conventional five parameter fit does not

\begin{tabular}{|c|c|c|c|c|c|}
\hline \multirow[b]{2}{*}{ Methods } & \multicolumn{5}{|c|}{ Parameters } \\
\hline & $\begin{array}{c}\text { Northern Cap } \\
\text { Albedo }\end{array}$ & $\begin{array}{c}\text { Northern Cap } \\
\text { Emissivity }\end{array}$ & $\begin{array}{c}\text { Southern Cap } \\
\text { Albedo }\end{array}$ & $\begin{array}{c}\text { Southern Cap } \\
\text { Emissivity }\end{array}$ & $\begin{array}{c}\text { Total Mass of } \mathrm{CO}_{2} \\
\text { in System } \\
\end{array}$ \\
\hline Positive perturbation constant weighting $\left(\mathbf{A}_{4,1}^{+}\right)$ & 0.796 & 0.484 & 0.467 & 0.787 & 0.978 \\
\hline Negative perturbation constant weighting $\left(\mathbf{A}_{4,1}^{-}\right)$ & 0.793 & 0.485 & 0.454 & 0.784 & 0.978 \\
\hline Average, constant weighting $\left(\boldsymbol{A}_{f 4,1}\right)$ & 0.795 & 0.485 & 0.461 & 0.785 & 0.978 \\
\hline Positive perturbation, nonconstant weighting $\left(\mathbf{A}_{4,2}^{+}\right)$ & 0.814 & 0.424 & 0.461 & 0.744 & 0.966 \\
\hline Negative perturbation, nonconstant weighting $\left(\mathbf{A}_{4,2}^{-}\right)$ & 0.820 & 0.434 & 0.433 & 0.764 & 0.971 \\
\hline Average, nonconstant weighting $\left(\boldsymbol{A}_{f 4,2}\right)$ & 0.817 & 0.429 & 0.447 & 0.754 & 0.968 \\
\hline
\end{tabular}
provide seasonal cap albedos and emissivities consistent

Table 1. Fitting Parameters for Different Perturbation Matrix and Time Weighting

${ }^{\mathrm{a}}$ Mass index number, 1.0 corresponds to total mass $2.90 \times 10^{16} \mathrm{~kg}$ of the baseline case. 

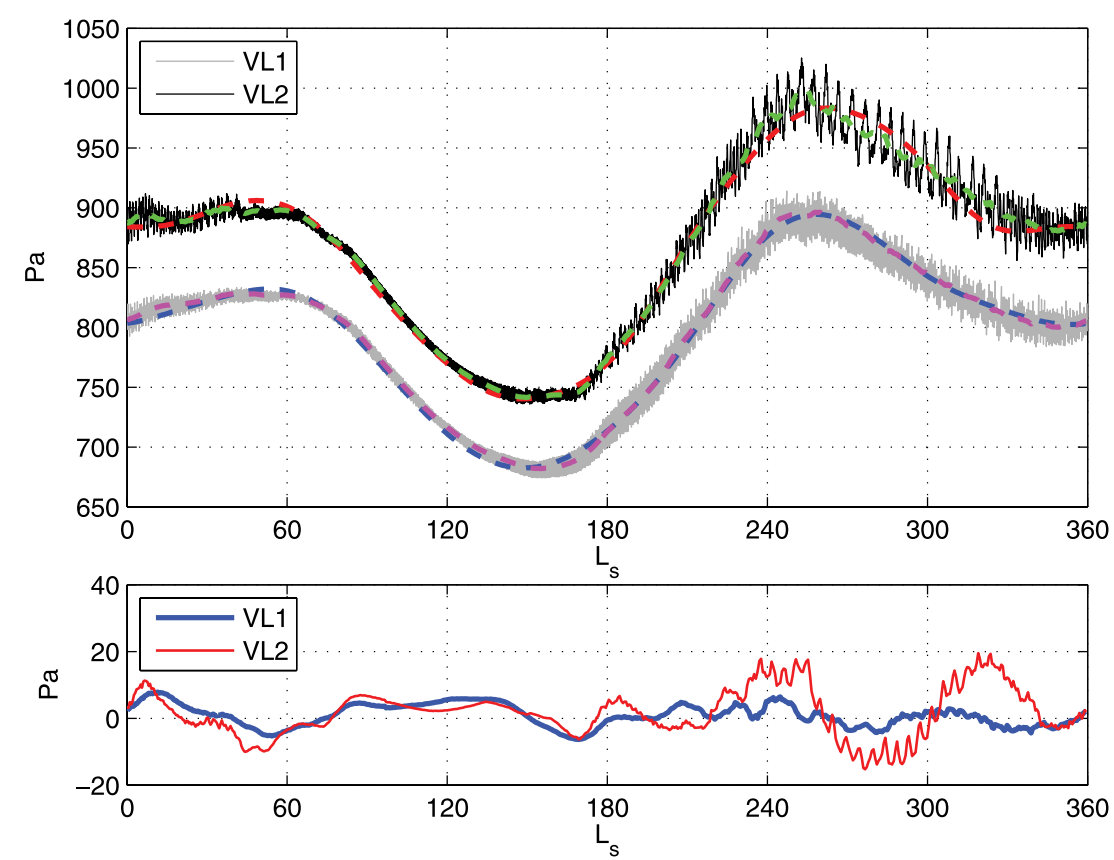

Figure 5. Same as Figure 1, except MarsWRF outputs are generated using the best fit parameters (northern seasonal cap albedo, 0.795 ; northern seasonal cap emissivity, 0.485; south albedo, 0.461; south emissivity, 0.785; total $\mathrm{CO}_{2}$ mass index, 0.978).

with prior observations, especially in the north, we try to improve the physics picture by including the subsurface layer thermal property as fitting parameters. As demonstrated in section 2.3 , sensitivity studies show that the water ice table thermal inertia has a similar footprint to that of the emissivity. On the other hand, it does not project a change in the surface pressure cycle that is as "linear" as that of emissivity. However, as long as the perturbation is not too large, we can still assume that the linear relationship holds.

[43] First, we try to extend the parameter vector to seven dimensions by adding the thermal inertia of the water ice table in the two hemispheres to the parameter vector. We extend the baseline parameter by two more dimensions as well and rewrite the baseline parameter vector as

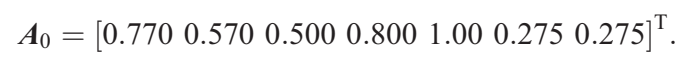

[44] The first five dimensions are defined as before. The sixth and seventh dimensions correspond to the thermal inertia of the water ice table in the north and south. In the baseline case, the subsurface is completely filled with dry soil. Therefore, the thermal inertia of the water ice table is assumed to be $275 \mathrm{~J} \mathrm{~m}^{-3} \mathrm{~K}^{-1}$, which is a value for average dry Martian soil. The perturbation vector $\mathbf{P}$ is also extended to

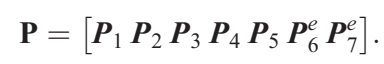

[45] Note that $\boldsymbol{P}_{6}^{e}$ and $\boldsymbol{P}_{7}^{e}$ were calculated with a different baseline value of the water ice table thermal inertia. In order to perform the linear fitting, we need to assume that these two pressure perturbation vectors do not change regardless of the baseline value. Undertaking a separate sensitivity study, we find that this is a reasonable assumption for the baseline values of 275 and $1200 \mathrm{~J} \mathrm{~m}^{-3} \mathrm{~K}^{-1}$, but later in this section we will examine a case in which this assumption needs to be reevaluated. Therefore, we use the same linear fitting method and obtain a new best fit parameter vector

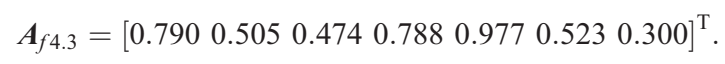

[46] This parameter vector has no essential difference to what we obtain from the conventional five-dimensional fit $\left(\boldsymbol{A}_{f 4.1}\right)$. The corrections to the parameters are just a few thousandths. When validated with MarsWRF, the residual pattern is very similar to before with slightly higher RMS error (about 3.97 Pa). It suggests that including a water ice table thermal inertia as fitting parameters will not produce a great improvement in the fitting. Thus, the conventional five-dimensional fit is sufficient for most atmospheric studies, in which the important thing is to obtain a surface pressure cycle that is within instrument error, while the accuracy of the retrieved frost radiative properties are considered less relevant (and indeed, obviously incorrect values can be tolerated, on the assumption that nonmodeled physics are aliased in these values).

[47] As discussed by Haberle et al. [2008], the significance of including subsurface ice is in a desire to gain physical consistency, i.e., being able to fit the VL pressure cycles with reasonable cap properties close to their observed values. We setup an experiment using unity frost emissivity, relatively small frost albedos, and subsurface water ice with fixed uniform depths to the water ice table (Figure 2) as assumed by Haberle et al. [2008]. We find that we are able to reproduce a pressure cycle reasonably close to that mea- 

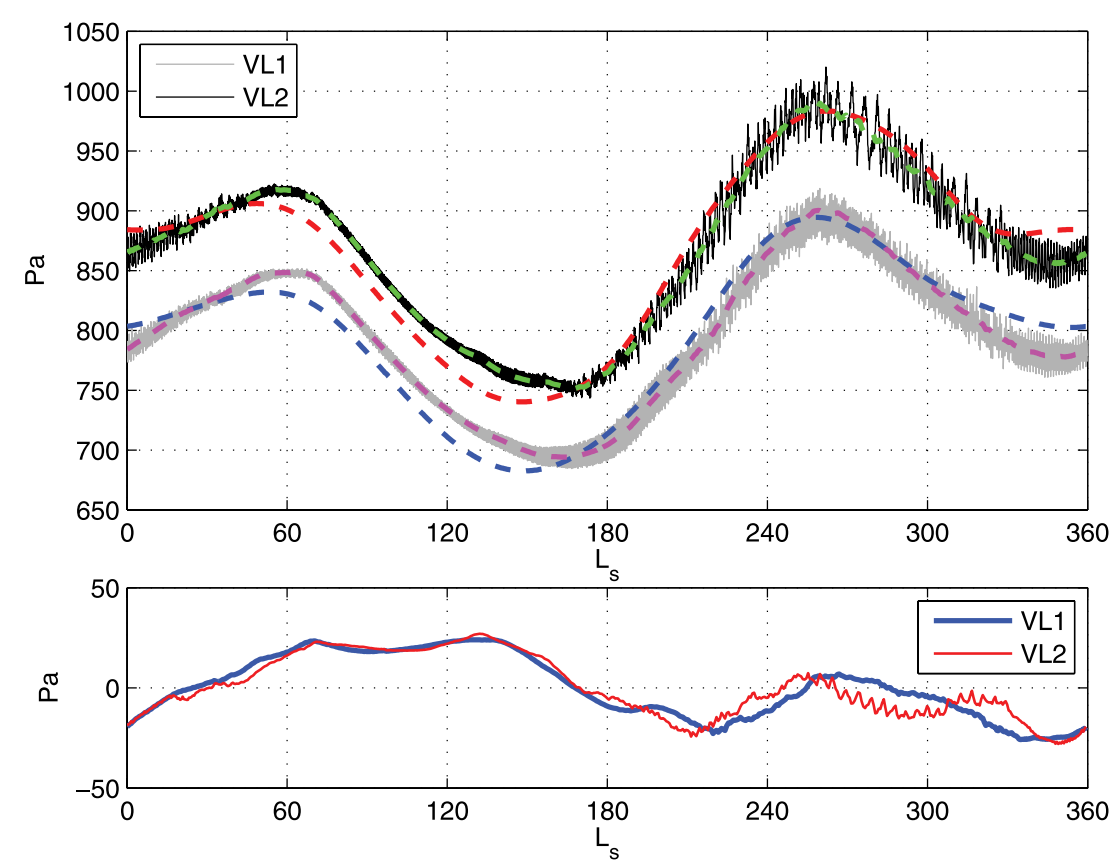

Figure 6. Same as Figure 1, except fitting parameter are the same as Haberle et al. [2008]. Northern seasonal cap albedo, 0.6; northern seasonal cap emissivity, 1.0; south albedo, 0.5; south emissivity, 1.0; total $\mathrm{CO}_{2}$ mass index, 1.003 . Water ice table starts at $8.05 \mathrm{~cm}$ in the northern hemisphere and $11.16 \mathrm{~cm}$ in the southern hemisphere. Thermal inertia of ice table is $2200 \mathrm{~J} \mathrm{~m}^{-3} \mathrm{~K}^{-1}$.

sured by VL1 (Figure 6, consistent with prior "by-eye" fitting but not as close as our five-parameter retrieval). While the amplitude of the cycle agrees well with the observation, the major discrepancy between the simulation and data is in the first half of the year, where the phases of the two curves do not perfectly agree. The same mismatch in phase can be found in the NASA Ames Research Center (ARC) Mars GCM simulations.

[48] The depth of the ice table will not in general be uniform in latitude or longitude, yet its depth is evidently readily predicted by simple 1-D water exchange models [Mellon and Jakosky, 1993; Schorghofer and Aharonson, 2005]. Following the philosophy of trying to find the most physically reasonable fit parameters, we decided to employ latitudinally varying depths of the water ice table as derived by Schorghofer and Aharonson [2005]. When we use these depths values and reduce the thermal inertia of the water ice tables from $2200 \mathrm{~J} \mathrm{~m}^{-3} \mathrm{~K}^{-1}$, we are also able to reproduce a reasonable fit to the VL pressure cycles (Figure 7). The quality of the fit is similar to that using the aforementioned ARC GCM setup, with the same phase mismatch during the northern summer. In this simulation, the water ice table top depends on latitude, and the thermal inertia assumed for the water ice table is not as high as pure water ice, with larger values for the north. If we also assume the same mixing ratio of water to dry soil in the water ice table, we find that the thermal inertia of the dry soil in the north is higher than the south, which agrees with IRTM and TES surface thermal inertia maps.

[49] Our sensitivity study has already shown that the seasonal cap emissivity and the water ice table thermal inertia have very similar footprints in the VL pressure cycle.
In other words, the role of emissivity in the linear fitting may be essentially replaced by the water ice table thermal inertia (and by extension, the emissivity from the conventional five-parameter fit may include aliased effects from neglected thermal inertia variations). Therefore, we proceed to do the numerical fitting with unity emissivity for the seasonal caps and instead vary the water ice table thermal inertia. In this case, the parameter vector still has five dimensions but the two dimensions corresponding to the seasonal cap emissivity are replaced by the thermal inertia of the water ice table in the north and south (their depth derived by Schorghofer and Aharonson [2005]). The best fit parameter vector thus retrieved is

$$
\boldsymbol{A}_{f 4.7}=\left[\begin{array}{lllll}
0.731 & 0.546 & 0.948 & 3.90 & 1.80
\end{array}\right]^{\mathrm{T}} .
$$

[50] The new fitting result is show in Figure 8. The RMS of the residual is now roughly $6.9 \mathrm{~Pa}$. When we use these parameters in MarsWRF, we are able to replicate the VL1 record very well (shown in Figure 9). The RMS of the error is $8.7 \mathrm{~Pa}$, slightly larger than with the prior five-parameter (and seven-parameter) fit, but satisfactory. No obvious phase disagreement between the two smoothed curves can be found.

[51] In summary, we succeed in fitting the VL data with the values of the albedos of the seasonal caps, the total $\mathrm{CO}_{2}$ inventory, and the subsurface layer thermal inertia, along with an assumption of unity seasonal cap emissivity. Although the fitting still retrieves an albedo of the northern seasonal cap higher than the south, the values are closer to the observation. The linear fitting results suggest that polar regolith with a 

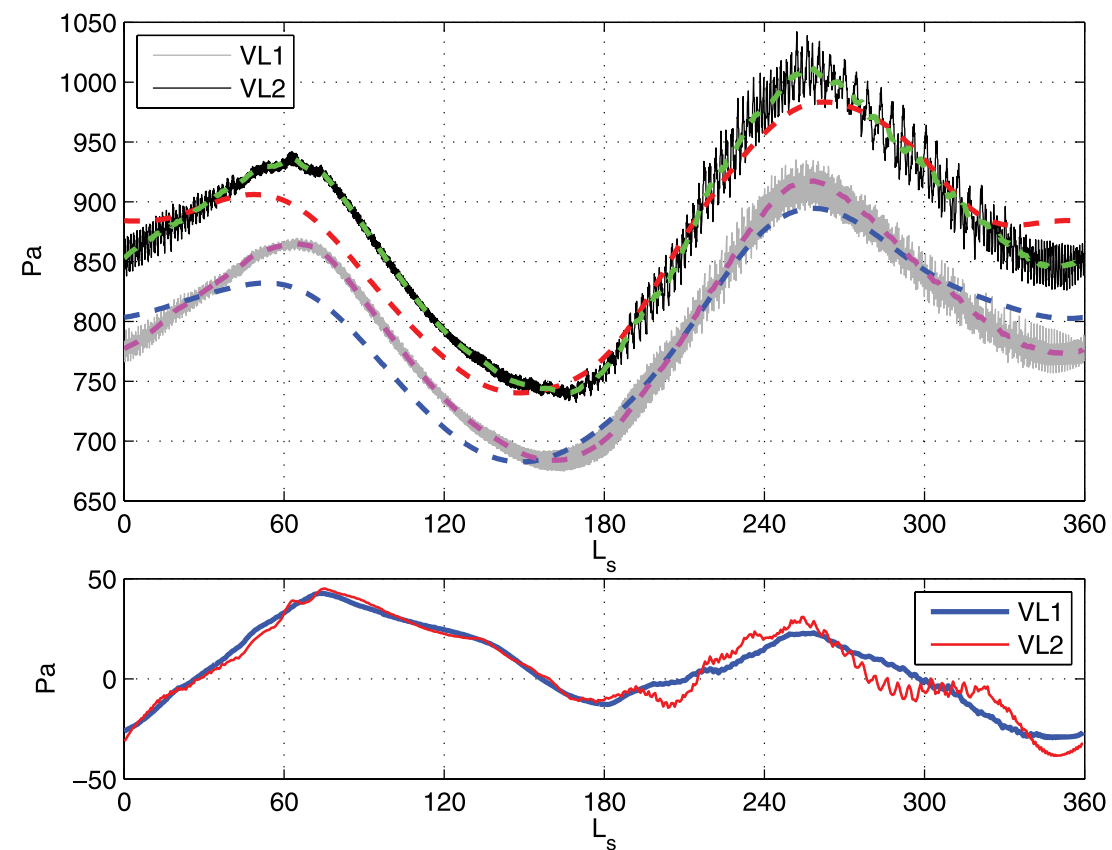

Figure 7. Same as Figure 1, except the driving parameters are as follows: northern seasonal cap albedo, 0.6; northern seasonal cap emissivity, 1.0; south albedo, 0.5 ; south emissivity, 1.0 ; total $\mathrm{CO}_{2}$ mass index, 1.003; thermal inertia of the ice table is $1800 \mathrm{~J} \mathrm{~m}^{-3} \mathrm{~K}^{-1}$ in the northern hemisphere and $900 \mathrm{~J} \mathrm{~m}^{-3} \mathrm{~K}^{-1}$ in the south. Boundary between the dry soil and the water ice table is set to the boundary of permanently stable water ice suggested by Schorghofer and Aharonson [2005].

higher thermal conductivity (due to subsurface water ice) is able to represent the extra heat source in autumn and winter, that used to be provided by excessively low cap emissivity in studies prior to Haberle et al. [2008]. The best fit thermal inertia values are very different in the two hemispheres, seemingly necessary to account for the different pressure levels during northern and southern winters. The predicted thermal inertia of the northern water ice table is much larger than that of the south, suggesting some sort of dichotomy in
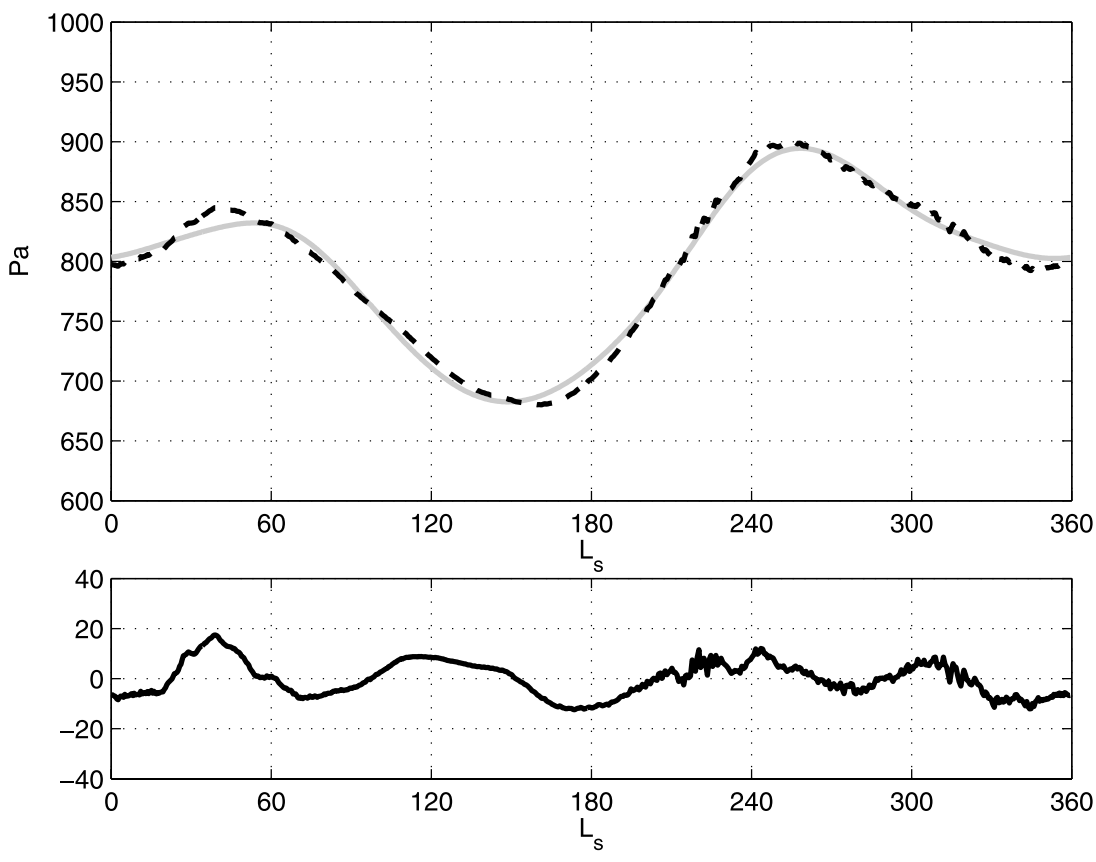

Figure 8. (top) Smoothed VL pressure cycle (gray line) and the fitted pressure curve (dash line) using albedo of the two seasonal caps, total $\mathrm{CO}_{2}$ inventory, and the thermal inertia of the water ice table at two different hemispheres. (bottom) Residual (fitted curve minus VL data). 

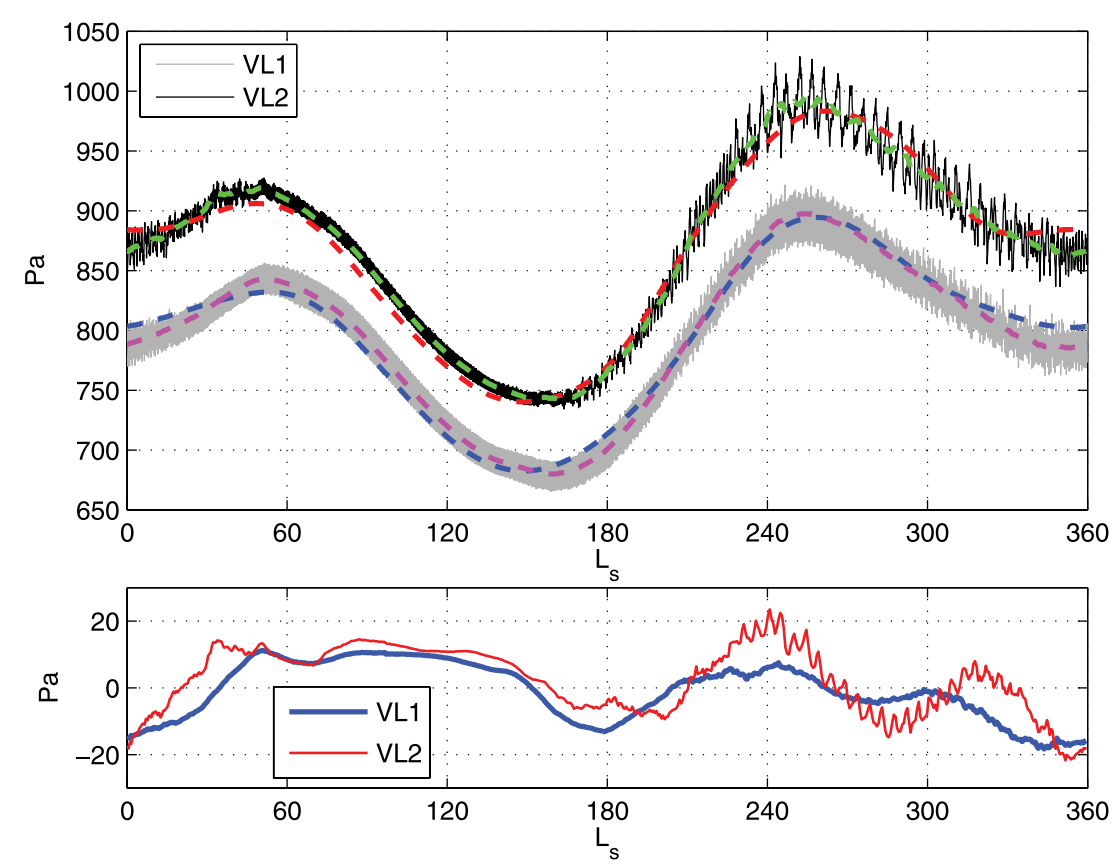

Figure 9. Same as Figure 7, except the driving parameters are as follows: northern seasonal cap albedo, 0.731; northern seasonal cap emissivity, 1.0 ; south albedo, 0.546 ; south emissivity, 1.0 ; total $\mathrm{CO}_{2}$ mass index, 1.077; thermal inertia of the ice table is $3900 \mathrm{~J} \mathrm{~m}^{-3} \mathrm{~K}^{-1}$ in the northern hemisphere and $1800 \mathrm{~J} \mathrm{~m}^{-3}$ $\mathrm{K}^{-1}$ in the south.

the thermal properties between the two hemispheres. We will discuss this issue in section 5 .

\section{Discussions of Parameterization}

\subsection{Albedo and Emissivity}

[52] Although albedo and emissivity may be modeldependent parameters, their physical interpretations are universal. By definition, albedo describes the fraction of solar energy reflected back to space while emissivity measures the fraction of energy in the blackbody curve for a surface of given kinetic temperature that is released from that emitter. If we first look at the retrievals without consideration of subsurface water ice, the five parameter linear fit suggests that the southern seasonal cap has lower albedo $(0.461)$ and higher emissivity $(0.785)$ than the north $(0.795$ and 0.485 , respectively). For these experiments, MarsWRF was not run in a mode to account for activities that change the effective albedo and emissivity (such as clouds, dust storms, etc.). Therefore, the best fit parameters not only characterize the seasonal caps but also provide hints as to other physical processes implicitly important to MarsWRF in the polar regions.

\subsubsection{Southern Seasonal Cap}

[53] When surface $\mathrm{CO}_{2}$ ice is mixed with dust, its albedo and emissivity change significantly. Assuming the same grain size of ice, a dustier deposit can produce a much less reflective and much more emissive surface material [Kieffer et al., 2000]. Hence, we expect that we can attribute many of the albedo and emissivity characteristics to the dust activity, which is usually stronger in the southern hemisphere [Basu et al., 2004; Martin, 1986; Smith, 2004]. However, it is less clear that increases in dust result in a generally darker and more emissive cap [James et al., 2000].
[54] Isotropic albedo (usually slightly higher than Lambert albedo) was mapped by TES [Kieffer et al., 2000]. The southern seasonal cap in general had albedos of 0.45 to 0.5 , with lower values (about 0.3 ) in the so-called "Cryptic regions" and higher values $(0.6$ to 0.7$)$ in the bright cap. On average, TES observations agree very well with the albedo predicted by the linear fit model for the south pole. Lambert albedo was also mapped by IRTM aboard the Viking orbiters. It ranged from 0.2 to 0.5 in the south [Paige and Keegan, 1994]. The region with an albedo 0.5 corresponded to the residual cap rather than the seasonal cap. Since the southern residual cap is believed to consist of mostly $\mathrm{CO}_{2}$ ice [Paige and Ingersoll, 1985], the IRTM observations for the $\mathrm{CO}_{2}$ cap are also consistent with our linear fit prediction. The Hubble Space Telescope has reported higher southern seasonal cap albedos for the bright cap region [James et al., 2005].

[55] We did not account for atmospheric $\mathrm{CO}_{2}$ cloud formation and precipitation with microphysical calculations in this version of MarsWRF (when the atmospheric temperature falls below the saturation point temperature for $\mathrm{CO}_{2}$ condensation, the model instantaneously deposits the corresponding condensed $\mathrm{CO}_{2}$ ice on the ground). It is believed that atmospheric $\mathrm{CO}_{2}$ condensation may affect the atmospheric state [Colaprete et al., 2008] and $\mathrm{CO}_{2}$ snowfall could contribute to the low brightness temperature or equivalently the low emissivity observed by various missions [Colaprete et al., 2005; Forget et al., 1999]. Some models have made efforts to relate the change in surface (seasonal cap) emissivity to the amount of condensed $\mathrm{CO}_{2}$. Forget et al. [1998] uses an empirical function to adjust the seasonal cap emissivity based on the atmospheric $\mathrm{CO}_{2}$ condensation and precipitation rate. Using equation (5) of that paper, we can calculate an average adjustment to the seasonal cap emissivity due to $\mathrm{CO}_{2}$ snow. For the southern cap, this correction is 


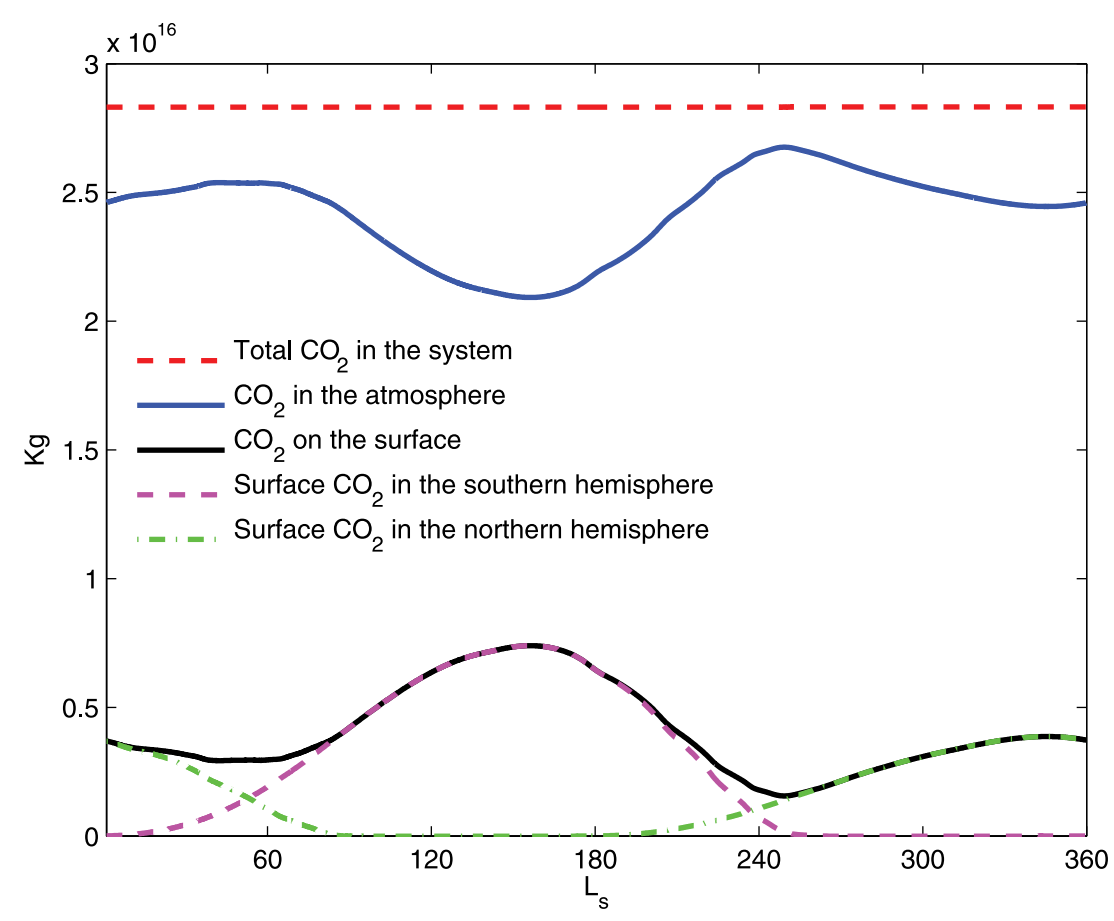

Figure 10. $\mathrm{CO}_{2}$ budget in MarsWRF using the best fit parameters (northern seasonal cap albedo, 0.795; northern seasonal cap emissivity, 0.485 ; south albedo, 0.461; south emissivity, 0.785 ; total $\mathrm{CO}_{2}$ mass index, 0.978). Red line, total $\mathrm{CO}_{2}$ in the system; blue line, mass of $\mathrm{CO}_{2}$ in the atmosphere; black line, mass of $\mathrm{CO}_{2}$ on the surface; magenta line, surface $\mathrm{CO}_{2}$ in the southern hemisphere; green line, surface $\mathrm{CO}_{2}$ in the northern hemisphere.

about 0.15 . When subtracted from the reference value of 0.95 , the effective emissivity is about 0.8 , which is very close to our best fit value. In other words, if $\mathrm{CO}_{2}$ snow is important, our time-constant parameterization may be effectively capturing this physical phenomenon while avoiding complexity in computation.

\subsubsection{Northern Seasonal Cap}

[56] Measurements of the northern seasonal cap albedo made by TES [Kieffer and Titus, 2001] are lower than our best fit value, and so are the IRTM observations [Paige et al., 1994; Paige and Keegan, 1994]. The polar hood cloud, which is usually seen in the winter time [James et al., 1992, 1994; Wang and Ingersoll, 2002], could contribute to the excessively high retrieved albedo of the northern cap. Furthermore, polar hood clouds are seen much less often in the southern hemisphere, and the retrieval results show that little correction is needed for the albedo of the southern cap, which starts relatively close to the observations. Although the radiative effects of hood clouds are not modeled by MarsWRF, the five-parameter set predicts the atmospheric mass budget for Mars very well. If hood cloud effects are significant, the retrieval scheme is effectively capturing (and aliasing) these effects into the northern seasonal cap retrieved albedo, by increasing the best fit albedo by 0.3 to 0.4 relative to the observations.

[57] When the effect of dust on the surface $\mathrm{CO}_{2}$ ice cap is ignored, emissivity can be considered as a function of the porosity of the $\mathrm{CO}_{2}$ frost: more porous ice tends to have lower emissivity [Eluszkiewicz et al., 2005]. Our best fit emissivity in the north is much lower than that in the south, suggesting a much more porous $\mathrm{CO}_{2}$ ice slab in the north. Porosity data of the two seasonal caps, which would provide a direct evalu- ation of our argument, are not yet available. However, our argument is at least consistent with the extremely low density of the northern seasonal cap [Aharonson et al., 2004].

[58] Finally, it should be noted that a good fit to the VL pressure data can still be obtained when the northern cap emissivity is assumed to be unity as long as subsurface water ice effects are included. Thus the degree to which the low northern emissivities must be explained is itself questionable.

\section{2. $\mathrm{CO}_{2}$ Inventory}

\subsubsection{Annual Variation}

[59] The global $\mathrm{CO}_{2}$ inventory cycle is plotted in Figure 10, divided into different reservoirs. Our best fit cases suggest that the total $\mathrm{CO}_{2}$ in the surface-atmosphere system is about $2.83 \times 10^{16} \mathrm{~kg}$, which agrees to within $5 \%$ of the NASA ARC GCM [Kelly et al., 2006]. $26 \%$ of the total $\mathrm{CO}_{2}(7.45 \times$ $10^{15} \mathrm{~kg}$ ) participates in the seasonal exchange between the atmosphere and the caps, slightly larger than the ARC GCM predictions, but consistent with earlier estimation [James et al., 1992; Kelly et al., 2006].

[60] As discussed in the sensitivity study, the amount of surface $\mathrm{CO}_{2}$ ice in MarsWRF is mainly determined by the albedo and emissivity of the corresponding seasonal caps. The prediction of surface $\mathrm{CO}_{2}$ in the southern hemisphere by MarsWRF matches closely with the GRS observations. In the north, our prediction is slightly higher than GRS observations [Kelly et al., 2006]. Ideally, GCMs should be able to simulate both surface $\mathrm{CO}_{2}$ deposition and VL site pressure cycles that agree well with the available observations, thus yielding an estimate of the total "active" $\mathrm{CO}_{2}$ budget in the system. Slight disagreements in simultaneously 
fitting the seasonal cap masses and lander pressure curves could result from interpolation errors given the relatively poor model resolution $\left(5^{\circ} \times 5.625^{\circ}\right)$, missing sources and sinks of $\mathrm{CO}_{2}$ (regolith), or instrument error.

\subsubsection{Permanent Polar Caps}

[61] A permanent $\mathrm{CO}_{2}$ cap exists at the Martian southern pole [Kieffer et al., 1977]. No published GCM predicts the existence of such a residual cap, and instead a residual cap must be prescribed in the model boundary conditions (often simply as a fixed surface temperature at the $\mathrm{CO}_{2}$ frost point). We have found that permanent $\mathrm{CO}_{2}$ caps will appear in MarsWRF only if extreme parameters (e.g., very high albedo values) are used, which agree with Wood and Paige [1992].

[62] The best fit parameter sets, which lead to good replications of the VL pressure cycle, do not produce permanent $\mathrm{CO}_{2}$ caps. This is not unexpected. Other GCMs do not produce permanent caps while trying to fit the VL pressure cycles [Haberle et al., 2008]. As stated in the introduction, Wood and Paige [1992] used a simple one dimension model for the thermal calculation to find the best parameter set to fit the VL pressure cycle. They pointed out that with their best fit values, the permanent cap is absent in both poles. We verified their conclusion once again using a much more sophisticated model (a GCM) that something "special" is happening in the heat balance at the south residual cap to somehow allow its existence. This is possibly due to an insolation-dependent albedo [James et al., 1992; X. Guo et al., On the mystery of the perennial carbon dioxide cap at the south pole of Mars, submitted to Journal of Geophysical Research, 2009].

\subsection{Subsurface Water Ice}

[63] As discussed in the sensitivity study, most of the subsurface water ice layer's footprint on the VL pressure cycle can be represented by a linear combination of the seasonal cap albedo and emissivity. Fitting using subsurface water ice (via the effects on subsurface conductivity) does not give particularly better fits. Rather, the advantage of fitting using surface ice is in retrieving cap properties that are more physically plausible. Subsurface water ice contributes directly to the seasonal energy balance, which is the driver of the $\mathrm{CO}_{2}$ annual cycle. Larger thermal conductivity provides the missing heat source in the energy balance in local winter, which before was represented by unrealistic frost albedo and emissivity. Its potential was already demonstrated in the GCM study by Haberle et al. [2008].

[64] The biggest difficulty in completing the picture is that we do not know precisely how deep the water ice is buried, what the water content is in the ice table, and how things change over time. The current assumptions in MarsWRF and ARC GCMs are more or less arbitrary. They are not backed up by many systematic observations, although the Phoenix lander observations do support the kind of ice table depth modeling undertaken by Mellon and Jakosky [1993] and Schorghofer and Aharonson [2005]. On the other hand, given very accurate measurements of the albedo and emissivity of the seasonal caps, we would likely be able to use the technique we have described to retrieve the (bulk) properties of subsurface water ice.

[65] The fit with $\mathrm{CO}_{2}$ albedo, total $\mathrm{CO}_{2}$ mass, and water ice table thermal inertia suggests much higher thermal conductivity for the subsurface layer in the north, to at least several meters below the surface. Since the spatial coverage of the water ice table is very different in the two hemispheres according to the GRS measurements (Figure 2), the difference in the conductive properties per unit mass of the regolith must be even larger. This is potentially a constraint on the nature of the Martian near-surface regolith. The crusts of the two hemispheres have long been known to differ [Neumann et al., 2004; Watters et al., 2007], so it may not be surprising to find they have different thermal conductive properties (or different abilities to accommodate highly conductive water ice).

[66] GRS reported hydrogen content and equivalent water content distributions (Figure 2) near the Martian surface [Feldman et al., 2004]. Assuming water ice occupies all the pores in the soil, and knowing the densities of water ice $\left(900 \mathrm{~kg} \mathrm{~m}^{-3}\right)$ and Martian crust $\left(2900 \mathrm{~kg} \mathrm{~m}^{-3}\right)$ [Zuber et al., 2000], we can back out the porosity of the soil. We make two more assumptions: first, the subsurface maintains the same porosity through the vertical column considered and that the pores are completely filled with water ice; second, the dry soil thermal conductivity in the subsurface is the same as the surface, whose equivalent thermal inertia is provided by TES [Putzig et al., 2005]. We can then calculate the thermal inertia of the subsurface mixture of soil and water ice (shown in Figure 11). An obvious north-south dichotomy in thermal inertia map can be seen. Zonal average subsurface thermal inertia ranges from 300 to $1400 \mathrm{~J} \mathrm{~m}^{-3} \mathrm{~K}^{-1}$ in the north, with a maximum of $1730 \mathrm{~J} \mathrm{~m}^{-3}$ $\mathrm{K}^{-1}$. In the south, the zonal average varies between 220 and $540 \mathrm{~J} \mathrm{~m}^{-3} \mathrm{~K}^{-1}$; this is on average about two to three times smaller than in the north. This dichotomy is due to the combined effects of high water ice content and high soil thermal inertia in the northern polar region. We notice that the values of thermal inertia obtained from this calculation are smaller than suggested by the retrieval scheme. There are ways to reduce this gap since the best fitted thermal inertia values could be misleading: MarsWRF assumes constant density and thermal capacity for the subsurface material. A decrease in dry soil density and/or heat capacity will decrease the thermal inertia while keeping the same thermal conductivity. One may also argue that the GRS may underpredict the water ice content and therefore the soil porosity. Setting those possibilities aside, there is an important common message from these two independent studies; that is, there exists a significant north-south dichotomy in the subsurface thermal conductivity in the polar regions.

[67] When we fix the seasonal cap emissivity to unity, we still need to adjust the seasonal cap albedos in order to make the seasonal pressure cycle phase match observations. This suggests that the inclusion of the water ice table, while improving physicality, is not the panacea - we do not yet have a complete and satisfactory understanding of the processes controlling the $\mathrm{CO}_{2}$ cycle. In order to build a better Mars GCM, we need to build more physically based prognostic models for the frost albedo and emissivity, capturing processes now implicitly aliased into our retrieved cap properties.

\subsection{Limitations of the Linear Fitting Method}

[68] As we discussed in section 3, the linear fitting algorithm assumes the response in surface pressure scales 


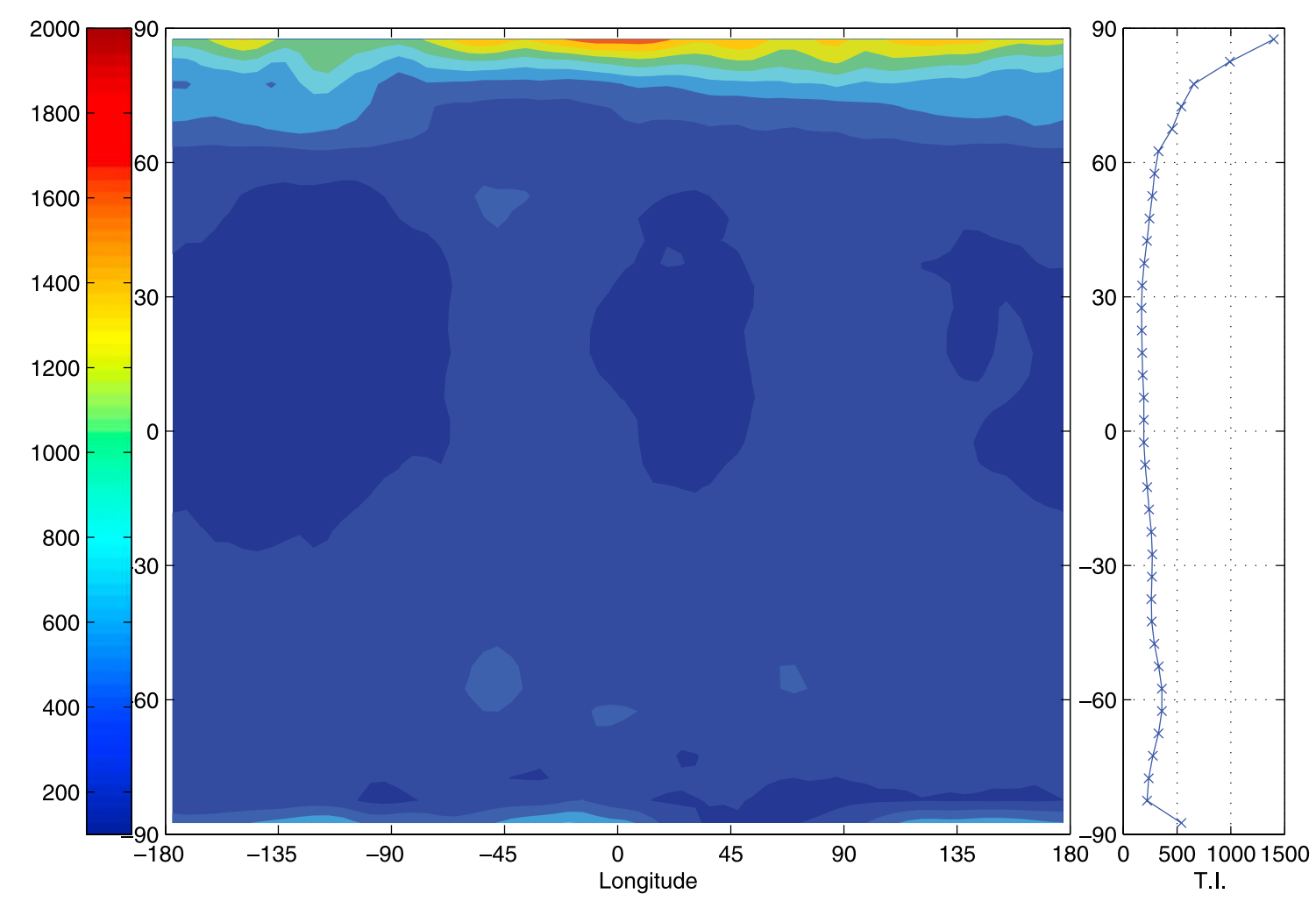

Figure 11. (left) Hypothesized subsurface thermal inertia in $\mathrm{J} \mathrm{m}^{-3} \mathrm{~K}^{-1}$ map and (right) zonal average.

linearly with the perturbation of the fitting parameters. In MarsWRF, this assumption becomes less valid when the perturbation is large, especially for perturbations to the water ice table thermal inertia. For example, in Figure 3 (bottom), when we perturb the thermal inertia baseline by 1000, 2000, and $3000 \mathrm{~J} \mathrm{~m}^{-3} \mathrm{~K}^{-1}$, the responses of the latter two are not simple multiples of the first. The perturbation matrix may change greatly when the baseline parameter vector moves to a different state. It may influence the calculation of the cost function and the iterative method: the last case from section 4.2 provides a good demonstration of these effects. If this is the case, we need to recalculate the pressure perturbation vectors to maintain linearity.

[69] The pattern of the perturbation matrix changes drastically in extreme situations. More specifically, MarsWRF does not respond to further change of parameters after critical values are reached. For instance, in Figure 3 (bottom), raising the water ice table thermal inertia by $3000 \mathrm{~J} \mathrm{~m}^{-3} \mathrm{~K}^{-1}$ does not increase the surface pressure much more than raising it by $2000 \mathrm{~J} \mathrm{~m}^{-3} \mathrm{~K}^{-1}$. In another situation where the frost albedo is too high, a permanent cap will form in the corresponding pole. One can easily imagine that the pressure response would have a very different pattern.

[70] Another related limitation of the iterative algorithm is that it is sensitive to the choice of the starting parameter vector. Starting the fitting with $\boldsymbol{Y}_{1}=\mathrm{F}\left(\boldsymbol{A}_{1}\right)$ may produce a different best fit parameter vector than starting with $\boldsymbol{Y}_{2}=$ $\mathrm{F}\left(\boldsymbol{A}_{2}\right)$, but both may yield comparable RMS errors. When used in MarsWRF, however, they may correspond to very different VL pressure cycles and RMS errors. Some are closer to the observations than the others. We have to choose the desired sets on the basis of their performances in the GCM and their physical soundness.
[71] Ideally, we should force the perturbations to be very small and recalculate the perturbation matrix for each iterative step. However, this would make the fitting extremely slow owing to the greater number of required iterations and, more importantly, more GCM runs to calculate the perturbation matrices. Fortunately, we can assume the perturbation matrix does not change for most of our retrievals. We also carefully selected the starting point of the iteration to make the process stable.

\section{Conclusion}

[72] We provide a method to obtain close fits to the VL surface pressure data using a chosen subset of "tunable" parameters within Mars General Circulation Models. We construct a set of basis vectors by perturbing five parameters in the GCM, including albedo and emissivity for the seasonal $\mathrm{CO}_{2}$ caps in both poles and the total amount of $\mathrm{CO}_{2}$ in the system. In this sense, our method is related to ensemble data assimilation. We utilize an iterative method to find the best linear combination of the basis vectors that minimizes the least squares error between the fit and the VL data. The coefficients for the linear fit are then projected back to parameter space and a best fit parameter set is obtained. When used in MarsWRF, this parameter set yields a pressure cycle very close to the VL observations as predicted by the linear method.

[73] The method described in this paper provides a first rigorous and quantitative means for fitting the seasonal pressure cycle with a GCM. As designed, we expect that this method would be useful for calibrating the $\mathrm{CO}_{2}$ cycle in any model and thus could provide a benchmark method for tuning GCMs. The study confirms the idea that a simple 
parameterization has the potential to capture most of the underlying physics of the $\mathrm{CO}_{2}$ cycle [Wood and Paige, 1992]. In the linear fit, the best fit parameters qualitatively show footprints of the physical processes that are not explicitly represented in MarsWRF but effectively captured by the parameterization, such as the presence of cloud, precipitation of atmospheric $\mathrm{CO}_{2}$ ice, surface frost with different porosity, etc.

[74] While the mathematics of the approach is elegant, the retrieved parameters are not guaranteed to be in agreement with direct observations. For instance, the cause of the big difference in the retrieved radiative properties of the two seasonal caps is not thoroughly understood. Attributing the differences to specific unmodeled physical process will require further work, along the philosophical lines laid out in the Introduction. However, our method provides a road map for the procedure of unveiling all relevant physics. We start with the simplest parameter set that fits the data well. Wherever the retrieved parameters differ from observed or physically sound values, we must look for further unresolved (unmodeled) physical processes. For example, parameterizations of clouds, dirty frost, etc., are likely still required. The soil thermal property change due to the presence of subsurface water ice is one such piece of physics we added in this study. Both MarsWRF and the NASA ARC Mars GCM have verified that including the subsurface ice improves the physical soundness of retrieved cap properties.

[75] Our simulations suggest that the northern subsurface layer has higher thermal conductivity than the south. This argument is also supported by GRS and TES observations. This bias in the inferred thermal conductivity may be related to differences in the water cycle, in the ability of the regolith materials in the two hemispheres to accommodate water, and/or in the regolith conductivity itself. Finally, none of the retrievals reproducing reasonable pressure curves are able to simultaneously yield a residual $\mathrm{CO}_{2}$ ice cap at the south. This may suggest that assumptions such as the temporally constant values of the retrieved cap properties may not be completely valid.

[76] Acknowledgments. We thank Oded Aharonson for the surface water content data, permanently stable water ice depth, and useful suggestions. We thank Andrew Ingersoll, Ashwin Vasavada, Stephen Wood, and Yuk Yung for helpful discussions. We thank two anonymous reviewers for their insightful comments. The numerical simulations for this research were performed on Caltech's Division of Geological and Planetary Sciences Dell cluster (CITerra).

\section{References}

Aharonson, O., M. T. Zuber, D. E. Smith, G. A. Neumann, W. C. Feldman, and T. H. Prettyman (2004), Depth, distribution, and density of $\mathrm{CO}_{2}$ deposition on Mars, J. Geophys. Res., 109, E05004, doi:10.1029 2003JE002223.

Basu, S., M. I. Richardson, and R. J. Wilson (2004), Simulation of the Martian dust cycle with the GFDL Mars GCM, J. Geophys. Res., 109, E11006, doi:10.1029/2004JE002243.

Boynton, W. V., et al. (2002), Distribution of hydrogen in the near surface of Mars: Evidence for subsurface ice deposits, Science, 297(5578), 81-85, doi:10.1126/science.1073722.

Colaprete, A., J. R. Barnes, R. M. Haberle, J. L. Hollingsworth, H. H. Kieffer, and T. N. Titus (2005), Albedo of the south pole on Mars determined by topographic forcing of atmosphere dynamics, Nature, 435, 184-188, doi:10.1038/nature03561.

Colaprete, A., J. R. Barnes, R. M. Haberle, and F. Montmessin (2008), $\mathrm{CO}_{2}$ clouds, CAPE and convection on Mars: Observations and general circulation modeling, Planet. Space Sci., 56(2), 150-180.
Eluszkiewicz, J., J. L. Moncet, T. N. Titus, and G. B. Hansen (2005), A microphysically based approach to modeling emissivity and albedo of the Martian seasonal caps, Icarus, 174(2), 524-534, doi:10.1016/ j.icarus.2004.05.025.

Feldman, W. C., et al. (2002), Global distribution of neutrons from Mars: Results from Mars Odyssey, Science, 297(5578), 75-78, doi:10.1126/ science. 1073541 .

Feldman, W. C., et al. (2004), Global distribution of near-surface hydrogen on Mars, J. Geophys. Res., 109, E09006, doi:10.1029/2003JE002160.

Forget, F., F. Hourdin, and O. Talagrand (1998), $\mathrm{CO}_{2}$ snowfall on Mars: Simulation with a general circulation model, Icarus, 131(2), 302-316, doi:10.1006/icar.1997.5874.

Forget, F., F. Hourdin, R. Fournier, C. Hourdin, O. Talagrand, M. Collins, S. R. Lewis, P. L. Read, and J. P. Huot (1999), Improved general circulation models of the Martian atmosphere from the surface to above $80 \mathrm{~km}$, J. Geophys. Res., 104(E10), 24,155-24,175, doi:10.1029/ 1999JE001025.

Guo, X., V. Natraj, D. R. Feldman, R. J. D. Spurr, R. Shia, S. P. Sander, and Y. Yung (2007), Retrieval of ozone profile from ground-based measurements with polarization: A synthetic study, J. Quant. Spectrosc. Radiat. Transfer, 103(1), 175-192, doi:10.1016/j.jqsrt.2006.05.008.

Haberle, R. M., F. Forget, A. Colaprete, J. Schaeffer, W. V. Boynton, N. J. Kelly, and M. J. Chamberlain (2008), The effect of ground ice on the Martian seasonal $\mathrm{CO}_{2}$ cycle, Planet. Space Sci., 56(2), 251-255, doi:10.1016/j.pss.2007.08.006.

Hess, S. L., R. M. Henry, C. B. Leovy, J. A. Ryan, and J. E. Tillman (1977), Meteorological results from the surface of Mars: Viking 1 and 2 , J. Geophys. Res., 82(28), 4559-4574, doi:10.1029/JS082i028p04559.

Hourdin, F., P. Levan, F. Forget, and O. Talagrand (1993), Meteorological variability and the annual surface pressure cycle on Mars, J. Atmos. Sci., 50(21), 3625-3640, doi:10.1175/1520-0469(1993)050<3625:MVATAS> 2.0.CO; 2 .

James, P. B., H. H. Kieffer, and D. A. Paige (1992), The seasonal cycle of carbon dioxide on Mars, in Mars, edited by H. H. Kieffer et al., pp. 934968, Univ. of Ariz. Press, Tucson.

James, P. B., R. T. Clancy, S. W. Lee, L. J. Martin, R. B. Singer, E. Smith, R. A. Kahn, and R. W. Zurek (1994), Monitoring Mars with the HubbleSpace-Telescope: 1990-1991 Observations, Icarus, 109(1), 79-101, doi:10.1006/icar.1994.1078

James, P. B., et al. (2000), The 1997 spring regression of the Martian south polar cap: Mars Orbiter camera observations, Icarus, 144(2), 410-418, doi:10.1006/icar.1999.6289.

James, P. B., B. P. Bonev, and M. J. Wolff (2005), Visible albedo of Mars' south polar cap: 2003 HST observations, Icarus, 174, 2, 596-599, doi:10.1016/j.icarus.2004.08.024.

Kelly, N. J., W. V. Boynton, K. Kerry, D. Hamara, D. Janes, R. C. Reedy, K. J. Kim, and R. M. Haberle (2006), Seasonal polar carbon dioxide frost on Mars: $\mathrm{CO}_{2}$ mass and columnar thickness distribution, J. Geophys. Res., 111, E03S07, doi:10.1029/2006JE002678.

Kieffer, H. H., and T. N. Titus (2001), TES mapping of Mars' north seasonal cap, Icarus, 154(1), 162-180, doi:10.1006/icar.2001.6670.

Kieffer, H. H., T. Z. Martin, A. R. Peterfreund, B. M. Jakosky, E. D. Miner, and F. D. Palluconi (1977), Thermal and albedo mapping of Mars during the Viking primary mission, J. Geophys. Res., 82(28), 4249-4291, doi:10.1029/JS082i028p04249.

Kieffer, H. H., T. N. Titus, K. F. Mullins, and P. R. Christensen (2000), Mars south polar spring and summer behavior observed by TES: Seasonal cap evolution controlled by frost grain size, J. Geophys. Res., 105(E4), 9653-9699, doi:10.1029/1999JE001136.

Martin, T. Z. (1986), Thermal infrared opacity of the Mars atmosphere, Icarus, 66(1), 2-21, doi:10.1016/0019-1035(86)90003-5.

Mellon, M. T., and B. M. Jakosky (1993), Geographic variations in the thermal and diffusive stablity of ground ice on Mars, J. Geophys. Res., 98(E2), 3345-3364.

Menemenlis, D., I. Fukumori, and T. Lee (2005), Using Green's functions to calibrate and ocean general circulation model, Mon. Weather Rev., 133, 1224-1240, doi:10.1175/MWR2912.1.

Mischna, M. A., J. F. Kasting, A. Pavlov, and R. Freedman (2000), Influence of carbon dioxide clouds on early Martian climate, Icarus, 145(2), 546-554, doi:10.1006/icar.2000.6380.

Neumann, G. A., M. T. Zuber, M. A. Wieczorek, P. J. McGovern, F. G. Lemoine, and D. E. Smith (2004), Crustal structure of Mars from gravity and topography, J. Geophys. Res., 109, E08002, doi:10.1029/ 2004JE002262.

Owen, T., K. Biemann, D. R. Rushneck, J. E. Biller, D. W. Howarth, and A. L. Lafleur (1977), The composition of the atmosphere at the surface of Mars, J. Geophys. Res., 82(28), 4635-4639, doi:10.1029/ JS082i028p04635.

Paige, D. A. (1992), The thermal stability of near-surface ground ice on Mars, Nature, 356, 43-45, doi:10.1038/356043a0. 
Paige, D. A., and A. P. Ingersoll (1985), Annual heat balance of the Martian polar caps: Viking observations, Science, 228(4704), 1160-1168, doi:10.1126/science.228.4704.1160

Paige, D. A., and K. D. Keegan (1994), Thermal and albedo mapping of the polar regions of Mars using Viking thermal mapper observations: 2. South polar region, J. Geophys. Res., 99(E12), 25,993-26,013, doi:10.1029/93JE03429.

Paige, D. A., J. E. Bachman, and K. D. Keegan (1994), Thermal and albedo mapping of the polar regions of Mars using Viking thermal mapper observations: 1. North polar region, J. Geophys. Res., 99(E12), 25,959-25,991, doi:10.1029/93JE03428.

Pollack, J. B., R. M. Haberle, J. R. Murphy, J. Schaeffer, and H. Lee (1993), Simulations of the general-circulation of the Martian atmosphere: 2. Seasonal pressure variations, J. Geophys. Res., 98(E2), 3149-3181, doi:10.1029/92JE02947.

Putzig, N. E., M. T. Mellon, K. A. Kretke, and R. E. Arvidson (2005), Global thermal inertia and surface properties of Mars from the MGS mapping mission, Icarus, 173(2), 325-341, doi:10.1016/j.icarus 2004.08.017.

Richardson, M. I., and R. J. Wilson (2002), A topographically forced asymmetry in the Martian circulation and climate, Nature, 416, $298-$ 301, doi:10.1038/416298a.

Richardson, M. I., R. J. Wilson, and A. V. Rodin (2002), Water ice clouds in the Martian atmosphere: General circulation model experiments with a simple cloud scheme, J. Geophys. Res., 107(E9), 5064, doi:10.1029/ 2001JE001804.

Richardson, M. I., A. D. Toigo, and C. E. Newman (2007), PlanetWRF: A general purpose, local to global numerical model for planetary atmospheric and climate dynamics, J. Geophys. Res., 112, E09001, doi:10.1029/2006JE002825.

Schorghofer, N., and O. Aharonson (2005), Stability and exchange of subsurface ice on Mars, J. Geophys. Res., 110, E05003, doi:10.1029/ 2004JE002350.
Smith, M. D. (2002), The annual cycle of water vapor on Mars as observed by the Thermal Emission Spectrometer, J. Geophys. Res., 107(E11), 5115, doi:10.1029/2001JE001522.

Smith, M. D. (2004), Interannual variability in TES atmospheric observations of Mars during 1999-2003, Icarus, 167(1), 148-165, doi:10.1016/ j.icarus.2003.09.010.

Tillman, J. E. (1988), Mars global atmospheric oscillations: Annually synchronized, transient normal mode oscillations and the triggering of global dust storms, J. Geophys. Res., 93(D8), 9433-9451, doi:10.1029/ JD093iD08p09433.

Tillman, J. E., N. C. Johnson, P. Guttorp, and D. B. Percival (1993), The Martian annual atmospheric pressure cycle: Years without great dust storms, J. Geophys. Res., 98(E6), 10,963-10,971, doi:10.1029/ 93JE01084.

Wang, H., and A. P. Ingersoll (2002), Martian clouds observation by Mars Global Surveyor Mars Orbiter Camera, J. Geophys. Res., 107(E10), 5078, doi:10.1029/2001JE001815.

Watters, T. R., P. J. McGovern, and R. P. Irwin III (2007), Hemispheres apart: The crustal dichotomy on Mars, Annu. Rev. Earth Planet. Sci., 35 , 621-652, doi:10.1146/annurev.earth.35.031306.140220.

Wood, S. E., and D. A. Paige (1992), Modeling the Martian seasonal CO cycle. 1. Fitting the Viking lander pressure curves, Icarus, 99(1), 1-14, doi:10.1016/0019-1035(92)90166-5.

Zuber, M. T., et al. (2000), Internal structure and early thermal evolution of Mars from Mars Global Surveyor topography and gravity, Science, 287(5459), 1788-1793, doi:10.1126/science.287.5459.1788

X. Guo, W. G. Lawson, and M. I. Richardson, Division of Geological and Planetary Sciences, California Institute of Technology, 1200 East California Boulevard, MC150-21, Pasadena, CA 91125, USA. (xin@gps.caltech.edu) A. Toigo, Center for Radiophysics and Space Research, Cornell University, Ithaca, NY 14850, USA. 